\title{
Topological defects in the Georgi-Machacek model
}

\author{
Chandrasekar Chatterjee, ${ }^{*}$ Masafumi Kurachi, ${ }^{\dagger}$ and Muneto Nitta \\ Department of Physics, and Research and Education Center for Natural Sciences, Keio University, \\ Hiyoshi 4-1-1, Yokohama, Kanagawa 223-8521, Japan
}

(Received 8 February 2018; published 8 June 2018)

\begin{abstract}
We study topological defects in the Georgi-Machacek model in a hierarchical symmetry breaking in which extra triplets acquire vacuum expectation values before the doublet. We find a possibility of topologically stable non-Abelian domain walls and non-Abelian flux tubes (vortices or cosmic strings) in this model. In the limit of the vanishing $U(1)_{Y}$ gauge coupling in which the custodial symmetry becomes exact, the presence of a vortex spontaneously breaks the custodial symmetry, giving rise to $S^{2}$ NambuGoldstone (NG) modes localized around the vortex corresponding to non-Abelian fluxes. Vortices are continuously degenerated by these degrees of freedom, thereby called non-Abelian. By taking into account the $U(1)_{\mathrm{Y}}$ gauge coupling, the custodial symmetry is explicitly broken, the NG modes are lifted to become pseudo-NG modes, and all non-Abelian vortices fall into a topologically stable $Z$ string. This is in contrast to the standard model in which $Z$ strings are nontopological and are unstable in the realistic parameter region. Non-Abelian domain walls also break the custodial symmetry and are accompanied by localized $S^{2}$ NG modes. Finally, we discuss the existence of domain wall solutions bounded by flux tubes, where their $S^{2} \mathrm{NG}$ modes match. The domain walls may quantum mechanically decay by creating a hole bounded by a flux tube loop, and would be cosmologically safe. Gravitational waves produced from unstable domain walls could be detected by future experiments.
\end{abstract}

DOI: 10.1103/PhysRevD.97.115010

\section{INTRODUCTION}

The standard model (SM) had been established as a reasonable low-energy effective description of the elementary particle physics, and its reliability has further improved since the discovery of the Higgs boson at the LHC [1,2]. However, the SM itself has theoretical problems, including a hierarchy problem, and it does not explain various things such as the neutrino mass, baryon asymmetry, dark matter, etc. These shortcomings of the SM might originate from its Higgs sector. Since the Higgs sector of the SM is constructed in a minimal way to describe the electroweak symmetry breaking, it might be too simple to deal with those problems. This thought motivates us to study extended models of the Higgs sector. The GeorgiMachacek (GM) model [3,4] is one such model, in which a field with $(\mathbf{3}, \overline{\mathbf{3}})$ representation of $S U(2)_{\mathrm{L}} \times S U(2)_{\mathrm{R}}$ global symmetry is introduced in addition to the Higgs

\footnotetext{
*chandra@phys-h.keio.ac.jp

†urachi@keio.jp

‡nitta@phys-h.keio.ac.jp
}

Published by the American Physical Society under the terms of the Creative Commons Attribution 4.0 International license. Further distribution of this work must maintain attribution to the author(s) and the published article's title, journal citation, and DOI. Funded by SCOAP ${ }^{3}$. doublet. The model incorporates Majorana mass of neutrinos through the type-II seesaw mechanism. An interesting feature of the GM model is that the vacuum expectation value (VEV) of the bitriplet field can be arranged in such a way to preserve the diagonal (custodial) $S U(2)$ symmetry of $S U(2)_{\mathrm{L}} \times S U(2)_{\mathrm{R}}$ at tree level; therefore, the magnitude of the VEV of the bitriplet does not necessarily have to be taken much smaller than the doublet VEV for the consistency with electroweak $\rho$ parameter measurement. It was shown that even at loop level, the custodial symmetry breaking effect coming from the hypercharge interaction is under control [5]. Having additional scalars, including the doubly charged particle, the phenomenology of the GM model is quite rich, and studies for direct detection at hadron colliders [6-8] and $e^{+}-e^{-}$colliders [9-11] have been done extensively. The model can be also distinguished from the SM or other extended models by precision Higgs coupling measurement at future experiments [12]. Various extensions of the GM model have been studied including the supersymmetric version [13], with fields higher than the triplet representation [14], the one incorporating an extra singlet to address the dark matter [15], etc. It was also shown that the strong first order electroweak phase transition, which is necessary for successful electroweak baryogenesis, could be achieved depending on the parameter choice [16].

Since the Higgs sector is extended in a nontrivial way, not only the mass spectrum, but also the vacuum structure, 
is quite different from that of the SM. In this paper we discuss the possible existence of topological defects in the Higgs sector of the GM model. Topological objects such as monopoles, strings, and domain walls may appear when a symmetry group is spontaneously broken and there exist nontrivial topological numbers or homotopy groups of the vacuum manifold of the symmetry breaking. That is, $\pi_{n}(G / H) \neq 0$, when symmetry group $G$ is spontaneously broken down to its subgroup $H$. As is well known today, the existence of such topological objects in the early Universe may have cosmological consequences. Cosmic strings can be thought of as a reconciliation between particle physics and cosmology. In the very hot dense early Universe it is assumed that electroweak symmetry or other symmetry is restored. During the process of expansion and cooling down, the Universe would have acquired domain structures due to a phase transition. A variety of topological objects may have been generated due to this phase transition process due to the Kibble-Zurek mechanism [17,18], and may have disappeared by recombination after subsequent symmetry breakings or by other dynamical processes. The presence of such objects sometimes gives constraints on models of elementary particle physics.

The first example of topological vortices in field theory was found in the Abelian-Higgs model [19] similar to Abrikosov vortices in a superconductor [20]. Vortices exist whenever the vacuum manifold $G / H$ admits a nontrivial first homotopy group, $\pi_{1}(G / H) \neq 0$. There are plenty of other examples of topological objects in grand unified models such as an $S O(10)$ model where $\mathbb{Z}_{2}$ vortices can appear [21]. Vortices behave as cosmic strings in the context of cosmology. For a review on cosmic strings, see Refs. [22,23]. In the context of the SM there exist electroweak strings [24-30]. However, these strings are not topologically stable since the fundamental group of the vacuum manifold

$$
\frac{S U(2)_{\mathrm{L}} \times U(1)_{\mathrm{Y}}}{U(1)_{\mathrm{em}}} \simeq S^{3}
$$

is trivial: $\pi_{1}\left(S^{3}\right)=0$. When the non-Abelian gauge coupling is turned off, they become so-called semilocal strings which are stable in the type-I superconductor parameter region [29,31]. Among all string solutions, $Z$ strings, containing a flux of $Z_{\mu}$ particles, can have a parameter region where they become stable $[25,26,28]$. If $Z$ strings are stable, they are suggested to contribute to electroweak baryogenesis [32,33], but there is also an objection [34]. However, they are unstable in the realistic parameter region of the SM. Fermion zero modes on $Z$ strings were also discussed in Refs. [35-43], in which it was argued that these zero modes may destabilize $Z$ strings. Moreover, end points of strings are attached by a monopole or an antimonopole. Therefore, the $Z$ strings can quantum mechanically decay by a nucleation of a monopole-antimonopole pair and are therefore at most metastable in this sense even in the stable parameter region [44]. $Z$ strings ending on monopoles were suggested to generate primordial magnetic fields in cosmology $[45,46]$. Saddle point solutions corresponding to the monopole and antimonopole connected by a $Z$ string are known as sphalerons [47].

Other than monopoles and strings, there can exist domain walls or kinks when a discrete symmetry is spontaneously broken. Domain wall or kink configurations depend on one spatial direction, appearing as a partition between two different vacua during phase transitions. Particularly common kinks appearing in various physical systems are sine-Gordon kinks [48-50] discussed for a long time starting from condensed matter physics, such as Josephson junctions of two superconductors [51] to cosmology [52]. Stable domain wall solutions are cosmologically forbidden, and so any model with stable domain wall solutions is ruled out. However, we can have domain walls which are separated from vacuum by the finite energy barrier. In this case, domain walls can decay by nucleation of a hole, typically bounded by a closed string [17,23,53-56]. For instance, axion models have a cosmological domain wall problem when an axion string is attached by multiple domain walls, while they are free from that problem when it is attached by one domain wall [57].

In this paper, we find out that there exist similar nontrivial topological structures (domain walls and electroweak strings) in the GM model if we consider a hierarchical symmetry breaking of the symmetry group $G$ in two stages; namely, only the triplets obtain the VEV first; then the doublet obtains its VEV later. The opposite ordering allows only the same vacuum manifold with that of the SM in Eq. (1) and is not new. This kind of hierarchy in symmetry breaking scales may have occurred during expansion and cooling periods of the early Universe. Similarly to the SM we have an electroweak gauge symmetry group $S U(2)_{\mathrm{L}} \times U(1)_{\mathrm{Y}}$ which we denote by $G_{\mathrm{Y}}$. The global symmetry group of the potential is found to be a larger group $S U(2)_{\mathrm{L}} \times S U(2)_{\mathrm{R}}$ than the $\mathrm{SM}$, which is the same as the symmetry group of the Lagrangian if we ignore the $U(1)_{Y}$ gauge interaction. We denote this enlarged group by $G$ and then we have the full symmetry breaking of $G$ as

$$
\begin{aligned}
G & =S U(2)_{\mathrm{L}} \times S U(2)_{\mathrm{R}} \stackrel{\Phi_{v}}{\rightarrow} \\
H_{3} & =\mathbb{Z}_{2} \times S U(2)_{V} \stackrel{\Psi_{v}}{\rightarrow} H_{2}=S U(2)_{\mathrm{V}},
\end{aligned}
$$

where $\Phi_{v}$ and $\Psi_{v}$ are triplet and doublet fields which acquire nonzero VEVs during each symmetry breaking stage. Here $S U(2)_{\mathrm{V}}$ is the diagonal subgroup of $G$, known as the custodial symmetry which remains unbroken throughout. Then we consider the case when $U(1)_{\mathrm{Y}}\left[\subset S U(2)_{\mathrm{R}}\right]$ is gauged. In this case, $S U(2)_{\mathrm{R}}$ is explicitly broken by the gauge field interaction. We find the symmetry breaking structure as 


$$
\begin{aligned}
& G_{\mathrm{Y}}=S U(2)_{\mathrm{L}} \times U(1)_{\mathrm{Y}} \stackrel{\Phi_{v}}{\rightarrow} \\
& H_{3}^{\mathrm{Y}}=\mathbb{Z}_{2} \times U(1)_{\mathrm{em}} \stackrel{\Psi_{v}}{\rightarrow} H_{2}^{\mathrm{Y}}=U(1)_{\mathrm{em}} .
\end{aligned}
$$

Here $U(1)_{\mathrm{em}}$ is the electromagnetic gauge group. For both the above cases we find nontrivial homotopy groups as

$$
\pi_{0}\left(H_{3} / H_{2}\right)=\mathbb{Z}_{2}, \quad \pi_{1}\left(G / H_{3}\right)=\mathbb{Z}_{2},
$$

implying the existence of domain walls and vortices, respectively, at each stage.

More precisely, if we consider the simplest case of the symmetry breaking of $\mathrm{H}_{3} / \mathrm{H}_{2}$, we have a domain wall solution due to $\pi_{0}\left(H_{3} / H_{2}\right)=\mathbb{Z}_{2}$. We find that this system has stable non-Abelian sine-Gordon kink solutions [5861]. These solutions spontaneously break the custodial $S U(2)_{\mathrm{V}}$ symmetry to a $U(1)$ subgroup, generating NambuGoldstone (NG) modes known as orientational zero modes [62-64]. These are collective coordinates giving an orientation of the unbroken $U(1)$ group within $S U(2)_{\mathrm{V}}$ on the $\operatorname{coset} S^{2} \simeq S U(2)_{\mathrm{V}} / U(1)$. Stable domain wall solutions are cosmologically forbidden, and so in this sense the GM model (with the hierarchical symmetry breaking) could have been ruled out. However, this is not the case because of $\pi_{1}\left(G / H_{3}\right)=\mathbb{Z}_{2}$ supporting $\mathbb{Z}_{2}$ vortex solutions. It is known that the existence of vortices which bound a domain wall can make domain walls decay [44] as axion domain walls, as mentioned above.

The $\mathbb{Z}_{2}$ string was originally discussed by Nielsen and Olesen [19] in an $S U(2)$ gauge theory coupled with two adjoint scalars. The $\mathbb{Z}_{2}$ strings and more generally $\mathbb{Z}_{N}$ strings in non-Abelian gauge theories were discussed for instance in Refs. [65-68]. In the GM model, the string solution is similar to a $\mathbb{Z}_{2}$ string discussed there. However, the $\mathbb{Z}_{2}$ string that we construct here is found to be nonAbelian in the sense that it contains non-Abelian flux directed along generic direction inside an internal space in the absence of the $U(1)_{Y}$ gauging. ${ }^{1}$ In the limit of vanishing $U(1)_{\mathrm{Y}}$ gauge coupling of the hypercharge, these vortices spontaneously break the custodial $S U(2)_{\mathrm{V}}$ symmetry to a $U(1)$ subgroup inside the vortex. This generates a continuous degeneracy in the whole vortex solutions, described by NG modes living on the $S^{2} \simeq S U(2)_{\mathrm{V}} / U(1)$ as the same as the case of non-Abelian domain walls mentioned above. So the flux can be directed along any generic direction on $S^{2} \simeq S U(2)_{\mathrm{V}} / U(1)$. These kinds of NG modes are known as orientational moduli of a non-Abelian vortex.

Non-Abelian vortices and their non-Abelian orientational moduli have been investigated extensively in the literature in great detail in supersymmetric gauge theories [69-78] and color-flavor locked phase in dense QCD [79-92]. They

\footnotetext{
${ }^{1} \mathrm{~A}$ non-Abelian $\mathbb{Z}_{2}$-string with non-Abelian moduli was discussed in the case of $\mathcal{N}=1^{*}$ supersymmetric gauge theories in Ref. [68].
}

may play a crucial role in understanding the confinement mechanism and duality in non-Abelian gauge theories. The dual confinement which is known as a monopole-vortex complex where magnetic monopoles are confined by the attachment of flux tubes in hierarchical symmetry breaking is discussed in Refs. [71-73,78,93-99]. The dual confinement in dense QCD was discussed in Ref. [100].

In our case, a non-Abelian vortex is attached by a nonAbelian domain wall where both $S^{2}$ moduli match, in the presence of the VEVs of both the triplets and doublet. In the limit of the vanishing interaction between the triplets and doublet, these $S^{2}$ modes can be different, and in this case, actually the domain wall disappears and the total configuration is reduced to a global vortex.

In the presence of $U(1)_{\mathrm{Y}}$ gauging, the custodial $S U(2)_{\mathrm{V}}$ symmetry is explicitly broken, the NG modes become pseudo-NG modes, and consequently the degeneracy of vortex solutions on full $S^{2}$ is lost and is reduced to the north and south poles and the equator circle. The same mechanism was studied in dense QCD [86,87] and supersymmetric QCD [101]. In this case we find that there exist two kinds of string solutions. First is a topologically stable $Z$ string corresponding to the poles on $S^{2}$ for which $S U(2)_{\mathrm{L}}$ and $U(1)_{\mathrm{Y}}$ gauge fields are parallel. We should emphasize here that our $Z$ strings are topologically stable, in contrast to $Z$ strings in the $\mathrm{SM}^{2}$ Other than the $Z$ strings, we also find $W$ strings in which the $S U(2)_{\mathrm{L}}$ gauge fields are orthogonal to the $U(1)_{\mathrm{Y}}$ gauge field and naturally the flux of the $W$ strings consists of $W$ bosons and no contribution from $U(1)_{\mathrm{Y}}$. The $W$ strings have an $S^{1}$ degeneracy and live on the equator circle of the $S^{2} \simeq S U(2)_{\mathrm{V}} / U(1)$. The $W$ strings have higher energy than the $Z$ strings and are unstable to decay to a $Z$ string.

This paper is organized as follows. In Sec. II we briefly introduce the GM model first and then discuss full twostage symmetry breakings in two subsequent subsections. In Sec. III we derive domain wall solution and discuss its orientational zero modes. In Sec. IV when the only triplets develop VEVs, we find non-Abelian vortex solutions in the limit of the vanishing $U(1)_{Y}$ gauge coupling by constructing profile functions numerically. We also discuss orientational zero modes. We then switch on $U(1)_{\mathrm{Y}}$ gauge coupling and obtain $Z$ strings and $W$ strings. In Sec. V we discuss the most general solutions in the presence of VEVs of the doublet and triplets. In the decoupling limit of the doublet and triplets, the flux tube of the triplet is accompanied with a winding of the doublet component and becomes a global vortex. In the presence of interaction between the doublet and triplets, it becomes a domain wallvortex composite state where a domain wall is bounded by a vortex flux tube. We then discuss the quantum mechanical decay of a domain wall by creating a hole bounded by a

\footnotetext{
${ }^{2}$ There exist topologically stable global electroweak strings in the two-Higgs doublet model [102-104].
} 
vortex loop and estimate the decay rate. Section VI is devoted to a summary and discussion.

\section{SYMMETRY OF THE GEORGI-MACHACEK MODEL}

Let us start by reviewing the GM model first; then we discuss the hierarchical symmetry breaking in this section.

\section{A. The Georgi-Machacek model}

The scalar sector of the SM supports an $G=$ $S U(2)_{\mathrm{L}} \times S U(2)_{\mathrm{R}}$ accidental symmetry. Out of the full symmetry group $G$, the $S U(2)_{\mathrm{L}}$ and $U(1)_{\mathrm{Y}}$ inside $S U(2)_{\mathrm{R}}$ is gauged to produce mass of the weak gauge bosons. It can be shown that the diagonal generator of $S U(2)_{\mathrm{R}}$ can be defined as the hypercharge. In this case the doublet scalar $\psi^{T}=\left(\psi_{1}, \psi_{2}\right)$ is written in $(\overline{2}, 2)$ form as $\Psi=\left(\begin{array}{c}\psi_{2}^{*} \psi_{1}^{*} \\ -\psi_{1}^{*} \psi_{2}\end{array}\right)$ and it breaks the symmetry group $G$ generating mass to weak gauge bosons. In this process, the VEV of the doublet keeps the diagonal subgroup $S U(2)_{\mathrm{V}}$ unbroken, which is known as the custodial symmetry. This model can be generalized by adding more scalars in different representations by keeping the symmetry structure the same, at least locally. The GM model [3] is one such extension. In this case the extension can be done by using an extra complex triplet $\Phi^{T}=\left(\phi_{++}, \phi_{+}, \phi_{0}\right)$, which can be written in $(\overline{3}, 3)$ form with the help of another real triplet scalar $\zeta^{T}=\left(\zeta_{+}, \zeta_{0}, \zeta_{-}\right)$as

$\Phi(x)=\left(\phi_{c}, \zeta, \phi\right)=\left(\begin{array}{ccc}\phi_{0}^{*} & \zeta_{+} & \phi_{++} \\ -\phi_{+}^{*} & \zeta_{0} & \phi_{+} \\ \phi_{++}^{*} & \zeta_{-} & \phi_{0}\end{array}\right)$,

where $\phi_{c}=C_{3} \phi^{*}{ }^{3}$

We start with the Lagrangian density as

$$
\begin{aligned}
\mathcal{L}= & -\frac{1}{4} W_{\mu \nu}^{a}{ }^{2}-\frac{1}{4} B_{\mu \nu}^{2}+\frac{1}{2} \operatorname{Tr}\left(\mathcal{D}_{\mu} \Phi\right)^{\dagger} \mathcal{D}_{\mu} \Phi \\
& +\frac{1}{2} \operatorname{Tr}\left(\mathcal{D}_{\mu} \Psi\right)^{\dagger} \mathcal{D}_{\mu} \Psi-V(\Phi, \Psi),
\end{aligned}
$$

${ }^{3}$ The notation of the matrices is as follows:

$$
\begin{aligned}
T^{1} & =\frac{1}{\sqrt{2}}\left(\begin{array}{lll}
0 & 1 & 0 \\
1 & 0 & 1 \\
0 & 1 & 0
\end{array}\right), \quad T^{2}=\frac{1}{\sqrt{2}}\left(\begin{array}{ccc}
0 & -i & 0 \\
i & 0 & -i \\
0 & i & 0
\end{array}\right), \\
T^{3} & =\left(\begin{array}{ccc}
1 & 0 & 0 \\
0 & 0 & 0 \\
0 & 0 & -1
\end{array}\right), \quad \operatorname{Tr}^{a} T^{b}=2 \delta^{a b}, \\
C_{3} & =\left(\begin{array}{ccc}
0 & 0 & 1 \\
0 & -1 & 0 \\
1 & 0 & 0
\end{array}\right), \quad \tau^{a}=\frac{1}{2} \sigma^{a} .
\end{aligned}
$$

where $\quad W_{\mu \nu}^{a}=\partial_{\mu} W_{\nu}^{a}-\partial_{\nu} W_{\mu}^{a}+g_{\mathrm{W}} \epsilon^{a b c} W_{\mu}^{b} W_{\nu}^{c}, \quad B_{\mu \nu}=$ $\partial_{\mu} B_{\nu}-\partial_{\nu} B_{\mu}$ are the field strengths of the gauge fields of $S U(2)_{\mathrm{L}}$ and $U(1)_{\mathrm{Y}}$ gauge symmetry, respectively, and the covariant derivatives are defined by

$$
\begin{aligned}
& \mathcal{D}_{\mu} \Phi=\left(\partial_{\mu}-i g_{\mathrm{W}} W_{\mu}^{a} T^{a}\right) \Phi+i g_{\mathrm{Y}} \Phi B_{\mu} T^{3} \\
& \mathcal{D}_{\mu} \Psi=\left(\partial_{\mu}-i g_{\mathrm{W}} W_{\mu}^{a} \tau^{a}\right) \Psi+i g_{\mathrm{Y}} \Psi B_{\mu} \tau^{3}
\end{aligned}
$$

Here $g_{\mathrm{W}}$ and $g_{\mathrm{Y}}$ are the coupling constants of $S U(2)_{\mathrm{L}}$ and $U(1)_{\mathrm{Y}}$ gauge interactions, respectively, and $T^{a}$ and $\tau^{a}$ are the triplet and doublet representations, respectively, of the generators of the $S U(2)$ algebra (see footnote 3 ). The potential that serves our purpose can be expressed as

$$
\begin{aligned}
V(\Phi, \Psi)= & \lambda_{1}\left(\operatorname{Tr} \Phi^{\dagger} \Phi-3 v_{3}^{2}\right)^{2} \\
& +\lambda_{2}\left[3 \operatorname{Tr} \Phi^{\dagger} \Phi \Phi^{\dagger} \Phi-\operatorname{Tr}\left(\Phi^{\dagger} \Phi\right)^{2}\right] \\
& +\lambda_{3}\left(\operatorname{Tr} \Psi \Psi^{\dagger} \Psi-v_{2}^{2}\right)^{2} \\
& +\lambda_{4}\left(\operatorname{Tr} \Psi+\Psi \operatorname{Tr} \Phi^{\dagger} \Phi\right. \\
& \left.-2 \operatorname{Tr}\left(\Psi^{\dagger} \tau^{a} \Psi \tau^{b}\right) \operatorname{Tr}\left(\Phi^{\dagger} T^{a} \Phi T^{b}\right)\right) .
\end{aligned}
$$

Here we consider the parameter region $\lambda_{1}+\lambda_{3}>0$, $\lambda_{2}>0, \lambda_{4}>0$. We have written a minimum number of terms in the potential required to fulfil our purposes $[4,14]$.

Now let us discuss symmetry of the Lagrangian defined in Eq. (7). We first discuss symmetry of the potential, which is the same as that of the case in which the gauge coupling of $U(1)_{\mathrm{Y}}$ (hypercharge) is turned off, i.e., $g_{\mathrm{Y}}=0$. Later we discuss the effect of $U(1)_{\mathrm{Y}}$ gauging. The potential in Eq. (9) is invariant under an enlarged symmetry group,

$$
G_{0}=\frac{S U(2)_{\mathrm{L}} \times S U(2)_{\mathrm{R}}}{\left(\mathbb{Z}_{2}\right)_{\mathrm{V}}}
$$

To understand the action of $G_{0}$ over the fields, let us define any element in the pair as $g=\left(g_{\mathrm{L}}, g_{\mathrm{R}}\right)$ in the universal covering group

$$
G=S U(2)_{\mathrm{L}} \times S U(2)_{\mathrm{R}} .
$$

The action of the group elements on the triplets and doublet can be defined as

$$
\begin{array}{ll}
\Phi^{\prime}(x)=g_{\mathrm{L}}\left(T^{a}\right) \Phi(x) g_{\mathrm{R}}^{\dagger}\left(T^{a}\right), & g_{\mathrm{L} / \mathrm{R}}\left(T^{a}\right)=e^{i \alpha_{\mathrm{L} / \mathrm{R}}^{a} T^{a}}, \\
\Psi^{\prime}(x)=g_{\mathrm{L}}\left(\tau^{a}\right) \Psi(x) g_{\mathrm{R}}^{\dagger}\left(\tau^{a}\right), & g_{\mathrm{L} / \mathrm{R}}\left(\tau^{a}\right)=e^{i \alpha_{\mathrm{L} / \mathrm{R}}^{a} \tau^{a}},
\end{array}
$$

respectively. Then, $\left(\mathbb{Z}_{2}\right)_{\mathrm{V}}$ in the denominator in Eq. (10) is given by $\left(\mathbb{Z}_{2}\right)_{\mathrm{V}}=\{(1,1),(-1,-1)\}$, since this group does not act on these fields. The full center $\mathbb{Z}_{2} \times \mathbb{Z}_{2}$ of the symmetry group $G$ is fully unseen by the triplet field $\Phi$, while $\left(\mathbb{Z}_{2}\right)_{\mathrm{A}}=\{(1,1),(1,-1)\}$ acts on the doublet field $\Psi$ although it does not act on the triplet fields $\Phi$ (and it is 
spontaneously broken when the doublet acquires a VEV). ${ }^{4}$ Hereafter, we work with the universal covering group.

\section{B. Symmetry breaking}

In this paper our purpose is to introduce a hierarchical symmetry breaking of the full symmetry group $G$. So our intension is to break the symmetry in two stages, first by the triplet field $\Phi(x)$ and then by the doublet field $\Psi(x){ }^{5}$ The details of the symmetry breaking process, temperature dependence, and fine-tuning of parameters can be discussed elsewhere. In this paper we just assume the possibility of two-stage symmetry breaking and for this purpose we assume $v_{3}>v_{2}$. For technical reasons we keep $v_{3} \gg v_{2}$; however, for practical purposes this constraint may not be very strict.

Let us now understand the symmetry breaking in detail. $S U(2)_{\mathrm{L}}$ and $S U(2)_{\mathrm{R}}$ groups act on the triplet field $\Phi$ from left and right accordingly as described in Eq. (12). Now we introduce the triplet VEV as

$$
\Phi_{v}=v_{3}\left(\begin{array}{lll}
1 & 0 & 0 \\
0 & 1 & 0 \\
0 & 0 & 1
\end{array}\right)
$$

As it can be understood easily from Eq. (12), the diagonal group elements $(g, g)$ of $S U(2)_{\mathrm{L}}$ and $S U(2)_{\mathrm{R}}$ do not act on the VEV. So $\Phi_{v}$ breaks the symmetry group $S U(2)_{\mathrm{L}} \times$ $S U(2)_{\mathrm{R}}$ and keeps the diagonal subgroup $S U(2)_{\mathrm{V}}$ unbroken. Including a discrete group, the unbroken group $\mathrm{H}_{3}$ inside the universal covering group $G$ is found to be

$H_{3}=S U(2)_{\mathrm{V}} \times\left(\mathbb{Z}_{2}\right)_{\mathrm{A}}, \quad\left(\mathbb{Z}_{2}\right)_{\mathrm{A}}=\{(1,1),(1,-1)\}$.

The $\mathbb{Z}_{2}$ in Eq. (15) is one of the normal subgroups of the center.4. The existence of this $\mathbb{Z}_{2}$ in $H_{3}$ can be understood easily if we consider the action of elements on the doublets and triplets separately.

\footnotetext{
${ }^{4}$ The center of the symmetry group $G$ can be written as the Klein-4 group $V_{4}=\mathbb{Z}_{2} \times \mathbb{Z}_{2}$. Elements of the center can be expressed in pairs as $V_{4}=\{e=(1,1), a=(-1,-1), b=(1,-1)$, $c=(-1,1)\}$. This group has three normal subgroups and can be written as $M_{v}=\{e, a\}, M_{1}=\{e, b\}, M_{2}=\{e, c\}$. Any two of them are permutable complements to each other. So $V_{4}$ can also be written as an internal direct product of any two of the above subgroups. One of them, namely, $M_{v}$, is the center of $S U(2)_{\mathrm{V}}$, the diagonal subgroup of $G$.

${ }^{5}$ The VEVs of the fields are temperature dependent in reality and the mass term in the potential can be expressed as $C_{1}\left[\left(\frac{T}{T_{c_{1}}}\right)^{2}-1\right] \operatorname{Tr}\left(\Phi^{\dagger} \Phi\right)+C_{2}\left[\left(\frac{T}{T_{c_{2}}}\right)^{2}-1\right] \operatorname{Tr}(\Psi \dagger \Psi)$. As the Universe cools down, the temperature $(T)$ may have reached a value $T_{c_{1}}>T>T_{c_{2}}$ where only first transition could occur.
}

The vacuum manifold is found to be

$$
\frac{G}{H_{3}}=\frac{S U(2)_{\mathrm{L}} \times S U(2)_{\mathrm{R}}}{\left(\mathbb{Z}_{2}\right)_{\mathrm{A}} \times S U(2)_{\mathrm{V}}} \simeq \frac{S U(2)}{\mathbb{Z}_{2}} \simeq S O(3) \simeq \mathbb{R} P^{3} .
$$

Since $G$ is simply connected we may express the fundamental group as

$$
\pi_{1}\left(\frac{G}{H_{3}}\right) \simeq \pi_{0}\left(H_{3}\right)=\mathbb{Z}_{2}
$$

implying the existence of a $\mathbb{Z}_{2}$ string.

The $\mathbb{Z}_{2}$ in $H_{3}$ in Eq. (15) nontrivially acts on the doublet, and so it is broken when the doublet acquires a VEV during the second symmetry breaking. The invariance of the potential under the group $\mathrm{H}_{3}$ can be understood clearly once we insert the value of $\Phi_{v}$ into the potential. After setting the triplet field $\Phi$ in its vacuum value $\Phi=v_{3} \mathbf{1}_{3 \times 3}$ the potential for the $\Psi$ field is found to be

$$
\begin{aligned}
V\left(v_{3} \mathbf{1}_{3 \times 3}, \Psi\right)= & \lambda_{3}\left(\operatorname{Tr} \Psi \dagger-v_{2}^{2}\right)^{2} \\
& +2 \lambda_{4} v_{3}^{2}\left(2 \operatorname{Tr} \Psi \Psi-|\operatorname{Tr} \Psi|^{2}\right) .
\end{aligned}
$$

We then find that the doublet field takes the form

$$
\Psi_{v}= \pm \frac{v_{2}}{\sqrt{2}}\left(\begin{array}{ll}
1 & 0 \\
0 & 1
\end{array}\right)
$$

in the vacua. This confirms the existence of $\mathbb{Z}_{2}$ and its breaking.

\section{The effect of $U(1)_{Y}$ symmetry}

So far we discussed the symmetry of the potential and its breaking. However, when we introduce the $U(1)_{\mathrm{Y}} \in$ $S U(2)_{\mathrm{R}}$ as local symmetry, the structure of the symmetry breaking changes a little. In this case $S U(2)_{\mathrm{R}}$ is explicitly broken and we may write the full symmetry group $G_{Y}$ of the Lagrangian as

$$
G_{\mathrm{Y}}=S U(2)_{\mathrm{L}} \times U(1)_{\mathrm{Y}} .
$$

The VEV of the triplet fields $\Phi_{v}$ in Eq. (14) breaks $G_{\mathrm{Y}}$ to $H_{3}^{\mathrm{Y}}=\mathbb{Z}_{2} \times U(1)_{\mathrm{em}}$. Here $U(1)_{\mathrm{em}}$ is the gauge group of electromagnetic theory and is defined as a subgroup of the custodial symmetry group $S U(2)_{\mathrm{V}}$. The VEV of the doublet $\Psi_{v}$ in Eq. (19) breaks $H_{3}^{\mathrm{Y}}$ to $U(1)_{\mathrm{em}}$. The full symmetry breaking in two stages is expressed in Eq. (3). In this case the vacuum manifold of the first symmetry breaking is different from what we found previously; however the fundamental group remains the same,

$$
\frac{G_{\mathrm{Y}}}{H_{3}^{\mathrm{Y}}}=\frac{S U(2)_{\mathrm{L}} \times U(1)_{\mathrm{Y}}}{\mathbb{Z}_{2} \times U(1)_{\mathrm{em}}}, \quad \pi_{1}\left(\frac{G_{\mathrm{Y}}}{H_{3}^{\mathrm{Y}}}\right)=\mathbb{Z}_{2} .
$$


We see that vortices and domain walls can be constructed in this case also. The only difference is the existence of electromagnetism and consequences are discussed later.

The kinetic term of the scalar field is given as $\mathcal{D}_{\mu} \Phi=\left(\partial_{\mu}-i g_{\mathrm{W}} W_{\mu}^{a} T^{a}\right) \Phi+i g_{\mathrm{Y}} \Phi B_{\mu} T^{3}$. In this case we define the well-known $Z_{\mu}$ boson and $A_{\mu}$ electromagnetic gauge field as

$$
\begin{aligned}
& A_{\mu}=\sin \theta_{\mathrm{W}} W_{\mu}^{3}+\cos \theta_{\mathrm{W}} B_{\mu}, \\
& Z_{\mu}=\cos \theta_{\mathrm{W}} W_{\mu}^{3}-\sin \theta_{\mathrm{W}} B_{\mu},
\end{aligned}
$$

where $\cos \theta_{\mathrm{W}}=\frac{g_{\mathrm{W}}}{\sqrt{g_{\mathrm{W}}^{2}+g_{\mathrm{Y}}^{2}}}$ and $\sin \theta_{\mathrm{W}}=\frac{g_{\mathrm{Y}}}{\sqrt{g_{\mathrm{W}}^{2}+g_{\mathrm{Y}}^{2}}}$. Using this Eq. (8) can be expressed as

$$
\begin{aligned}
\mathcal{D}_{\mu} \Phi= & \left(\partial_{\mu}-i g_{\mathrm{W}} \sum_{ \pm} W_{\mu}^{ \pm} T^{ \pm}\right) \Phi \\
& -i Z_{\mu}\left(g_{\mathrm{W}} \cos \theta_{\mathrm{W}} T^{3} \Phi+g_{\mathrm{Y}} \sin \theta_{\mathrm{W}} \Phi T^{3}\right) \\
& -i g_{\mathrm{W}} \sin \theta_{\mathrm{W}} A_{\mu}\left(T^{3} \Phi-\Phi T^{3}\right) .
\end{aligned}
$$

So naturally we can define electric charge $e=\frac{g_{\mathrm{W}} g_{\mathrm{Y}}}{\sqrt{g_{\mathrm{W}}^{2}+g_{\mathrm{Y}}^{2}}}$. At the vacuum when first symmetry breaking occurs $\Phi=v_{3} \mathbf{1}$, the $A_{\mu}$ interaction vanishes. A similar situation occurs for the doublet also. After full symmetry breaking the masses of gauge fields are given by

$$
\begin{aligned}
m_{Z}^{2} & =\left(2 v_{3}^{2}+\frac{1}{4} v_{2}^{2}\right) g_{\mathrm{Z}}^{2}, \quad m_{W}^{2}=\left(2 v_{3}^{2}+\frac{1}{4} v_{2}^{2}\right) g_{\mathrm{W}}^{2}, \\
g_{\mathrm{Z}} & =\sqrt{g_{\mathrm{W}}^{2}+g_{\mathrm{Y}}^{2}}
\end{aligned}
$$

We are assuming the $v_{3}>v_{2}$ case so the masses are dominated by the VEV $v_{3}$ of the triplets.

\section{NON-ABELIAN DOMAIN WALLS}

The domain wall solution occurs whenever the discrete symmetries of a field theory are spontaneously broken in the ground state. In the case of the situation described above we saw that after the first phase transition which is triggered by the triplet VEV, our potential is invariant under a symmetry group $\mathrm{H}_{3}$ which contains a discrete subgroup of the original symmetry group $S U(2)_{\mathrm{L}} \times S U(2)_{\mathrm{R}}$ and described as $\mathbb{Z}_{2} \times S U(2)_{\mathrm{V}}$. Now if we observe the second phase transition as in Eq. (2), we may notice that it breaks $\mathbb{Z}_{2}$, implying the existence of a domain wall.

Let us first start with $g_{\mathrm{Y}}=0$, that is, without $B_{\mu}$ interaction. Since in this case all the existing gauge fields become massive after the first phase transition, we may ignore them while constructing domain walls. Hence, let us consider the following reduced Lagrangian constructed by inserting the vacuum expectation value of the triplet as $\Phi=v_{3} \mathbf{1}_{3 \times 3}$ into Eqs. (7) and (9) and also by setting all the gauge fields equal to 0 ,

$$
\begin{aligned}
\mathcal{L}_{2}= & \frac{1}{2} \operatorname{Tr}\left(\partial_{\mu} \Psi\right)^{\dagger} \partial_{\mu} \Psi-\lambda_{3}\left(\operatorname{Tr} \Psi \dagger \Psi-v_{2}^{2}\right)^{2} \\
& -2 \lambda_{4} v_{3}^{2}\left(2 \operatorname{Tr} \Psi \Psi-\operatorname{Tr} \Psi^{\dagger} \operatorname{Tr} \Psi\right) .
\end{aligned}
$$

Now we define our domain wall static ansatz as

$$
\Psi(x)_{\mathrm{dw}}=\frac{v_{2}}{\sqrt{2}} \psi(x) \exp \left[i \phi(x) \tau^{3}\right] .
$$

Here the fields $\psi(x)$ and $\phi(x)$ are functions of a single spacial coordinate assuming that the center of the wall is on the orthogonal plane at $x=0$. After inserting the ansatz into the Lagrangian we find

$$
\begin{aligned}
-\frac{\mathcal{L}_{2}}{v_{2}^{2}}= & \frac{1}{2}\left(\partial_{i} \psi\right)^{2}+\frac{1}{8} \psi^{2}\left(\partial_{i} \phi\right)^{2}+\lambda_{3} v_{2}^{2}\left(\psi^{2}-1\right)^{2} \\
& +2 \lambda_{4} v_{3}^{2} \psi^{2}(1-\cos \phi) .
\end{aligned}
$$

Now, to make the point clearer, let us consider the extreme situation when $\frac{\lambda_{3}}{\lambda_{4}} \gg \frac{v_{3}^{2}}{v_{2}^{2}}$. In this case we may assume $\psi(x)$ can be set at one of the vacua, say, $\psi(x)=1$; then we may have a domain wall in $\phi$ with the boundary conditions

$$
\phi(x=\infty)=2 \pi, \quad \phi(x=-\infty)=0,
$$

and this corresponds to $\mathbb{Z}_{2}$ transformation at the boundary as $\Psi(-\infty)^{\not /}-\Psi(\infty)$. In this case the Lagrangian reduces to

$-\frac{\mathcal{L}_{\phi}}{v_{2}^{2}}=\frac{1}{8}\left[\left(\partial_{i} \phi\right)^{2}+8 \mu^{2}(1-\cos \phi)\right], \quad \mu^{2}=2 \lambda_{4} v_{3}^{2}$.

This is identical to the sine-Gordon model and a domain wall solution interpolating between the two vacua can be written as

$$
\phi(x)=4 \tan ^{-1} e^{ \pm 2 \mu x},
$$

where the width of the domain wall is given by $\delta_{\mathrm{dw}} \sim \mu^{-1}$ and the energy per unit area can be written as

$$
T_{\mathrm{dw}}=4 \mu v_{2}^{2} .
$$

The shape of the solution is shown in Fig. 1.

Here we may say a few words on the solution ansatz in Eq. (26). As is discussed before, the vacuum after the second symmetry breaking preserves the $S U(2)_{\mathrm{V}}$ custodial symmetry. The presence of a domain wall configuration spontaneously breaks the $S U(2)_{\mathrm{V}}$ custodial symmetry into a $U(1)$ subgroup in the vicinity of the wall. It can be checked easily that at the boundary where $\phi=(0,2 \pi)$ the custodial symmetry is recovered. This spontaneous breaking of the custodial symmetry generates NG modes. These are orientational zero modes on the domain wall surface parametrizing the coset space $S^{2} \simeq S U(2)_{\mathrm{V}} / U(1)$. The 


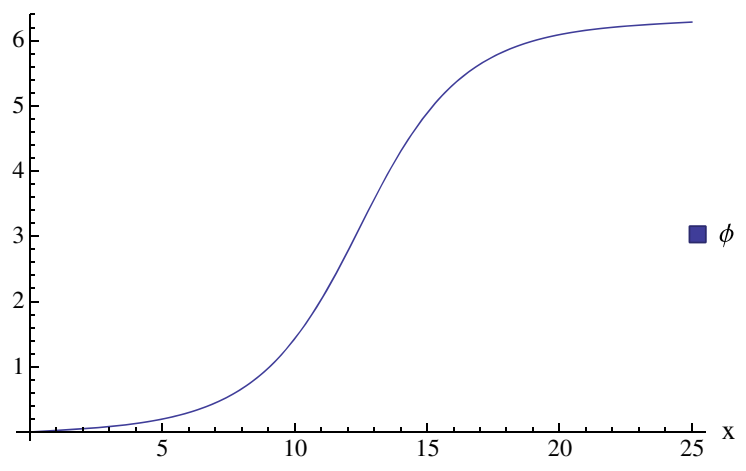

FIG. 1. A plot of the Sine-Gordon kink.

existence of these modes allows us to define the ansatz in generic direction on $S^{2}$ by a global transformation as

$$
\begin{aligned}
\Psi\left(\xi^{\alpha}, x\right) & =G\left(\xi^{\alpha}\right) \frac{v_{2}}{\sqrt{2}} \psi \exp \left[i \phi \tau^{3}\right] G^{\dagger}\left(\xi^{\alpha}\right) \\
& =\frac{v_{2}}{\sqrt{2}} \psi(x) \exp [i \phi(x) \hat{\mathbf{n}}]
\end{aligned}
$$

where $\hat{\mathbf{n}}=G\left(\xi^{\alpha}\right) \tau^{3} G^{\dagger}\left(\xi^{\alpha}\right)$,

$$
G\left(\xi^{\alpha}\right)=\left(\begin{array}{cc}
\cos \frac{\xi^{1}}{2} & -\sin \frac{\xi^{1}}{2} e^{-i \xi^{2}} \\
\sin \frac{\xi^{1}}{2} e^{i \xi^{2}} & \cos \frac{\xi^{1}}{2}
\end{array}\right),
$$

where $\xi^{\alpha}$ are the coordinate angles defined on $S^{2}$ and $\operatorname{tr}\left(\hat{\mathbf{n}}^{2}\right)=1$. The effective theory of the NG modes should be a $O(3)$ sigma model on the 2+1-dimensional world volume.

Now we should talk about the case when $g_{\mathrm{Y}} \neq 0$. Since domain wall construction depends only on scalar fields but not on gauge field interactions, the introduction of $U(1)_{\mathrm{Y}}$ would not effect the construction at tree level. The radiative correction breaks $S U(2)$ custodial symmetry explicitly, and hence $S^{2}$ moduli are lifted. Also, the presence of electromagnetic field generates interaction with $S^{2}$ zero modes. So the effective action of the static domain wall is described by an $S O(2)$ gauged $O(3)$ sigma model living in a $2+1$ dimensional hyperplane interacting with an electromagnetic gauge field living in $3+1$-dimensional space.

\section{NON-ABELIAN VORTICES AND TOPOLOGICAL $Z$ STRINGS}

In this section, we discuss vortices in the first symmetry breaking in which only the triplets acquire VEVs. In the first subsection, we discuss a non-Abelian vortex in the limit of the absence of the $U(1)_{Y}$ gauge interaction. In the second subsection, we discuss that non-Abelian vortices reduce to a $Z$ string or $W$ string when we turn on the $U(1)_{\mathrm{Y}}$ gauge interaction.

\section{A. Non-Abelian vortices in the absence of the $U(1)_{Y}$ gauge interaction}

Here we assume $g_{\mathrm{Y}}=0$ at the start and the effect of $g_{\mathrm{Y}} \neq 0$ is discussed in the next subsection. To construct vortices we only concentrate on the first phase transition as discussed in Eq. (2). Since $\pi_{1}\left(G / H_{3}\right)=\mathbb{Z}_{2}$, we may have a vortex solution. In this section, to avoid complication, we set the doublet field to 0 . The consequences of interaction with the doublet field are discussed in the next section.

So we start with the Lagrangian density

$$
\mathcal{L}=-\frac{1}{4} W_{\mu \nu}^{a}{ }^{2}+\frac{1}{2} \operatorname{Tr}\left(\mathcal{D}_{\mu} \Phi\right)^{\dagger} \mathcal{D}_{\mu} \Phi-V(\Phi)
$$

$V(\Phi)=\lambda_{1}\left(\operatorname{Tr} \Phi^{\dagger} \Phi-3 v_{3}^{2}\right)^{2}+\lambda_{2}\left[3 \operatorname{Tr} \Phi^{\dagger} \Phi \Phi^{\dagger} \Phi-\operatorname{Tr}\left(\Phi^{\dagger} \Phi\right)^{2}\right]$,

where $W_{\mu \nu}^{a}=\partial_{\mu} W_{\nu}^{a}-\partial_{\nu} W_{\mu}^{a}+g_{\mathrm{W}} \epsilon^{a b c} W_{\mu}^{b} W_{\nu}^{c}$ and $\mathcal{D}_{\mu} \Phi=$ $\left(\partial_{\mu}-i g_{\mathrm{W}} W_{\mu}^{a} T^{a}\right) \Phi$. Here $g_{\mathrm{W}}$ is the coupling constant of $S U(2)_{\mathrm{L}}$ gauge interactions.

For simplification we construct an infinitely long vortex along the $z$ axis with a cylindrical symmetry. To derive a vortex solution let us start with the ansatz of $\Phi$ and $W_{\mu}$ as

$$
\begin{aligned}
\Phi_{\text {vortex }} & =v_{3}\left(\begin{array}{ccc}
f(r) e^{i \theta} & 0 & 0 \\
0 & g(r) & 0 \\
0 & 0 & f(r) e^{-i \theta}
\end{array}\right), \\
W_{i}^{a} & =-\frac{\epsilon_{i j} x^{j}}{g_{\mathrm{W}} r^{2}}(1+h(r)) \delta^{a 3},
\end{aligned}
$$

where $i=1,2$ and $W_{0}^{a}$ and $W_{3}^{a}$ are taken to be 0 . Boundary conditions for profile functions are taken to be

$$
\begin{array}{cll}
f(0)=0, & f(\infty)=1, & g^{\prime}(0)=0, \\
g(\infty)=1, & h(0)=-1, & h(\infty)=0 .
\end{array}
$$

Here $(r, \theta)$ are the radius and azimuthal angle of the cylindrical coordinates. Let us first consider a large distance behavior of the vortex ansatz. From the above solution ansatz we may write

$$
\begin{aligned}
\Phi_{\text {vortex }}(\theta, \infty) & =v_{3}\left(\begin{array}{ccc}
e^{i \theta} & 0 & 0 \\
0 & 1 & 0 \\
0 & 0 & e^{-i \theta}
\end{array}\right)=\Omega(\theta) \Phi_{v}, \\
\text { where } \Omega & =e^{i \theta T_{3}} \in \frac{G}{H_{3}} .
\end{aligned}
$$

To find the behavior of the profile functions, let us just put the above ansatz in the potential in Eq. (35) to yield 


$$
\begin{aligned}
V(\Phi)= & \lambda_{1} v_{3}^{4}\left(2 f(r)^{2}+g(r)^{2}-3\right)^{2} \\
& +2 \lambda_{2} v_{3}^{4}\left[f(r)^{2}-g(r)^{2}\right]^{2} .
\end{aligned}
$$

The static Hamiltonian density

$$
\mathcal{H}=\int d^{2} x\left[\frac{1}{4} W_{i j}^{a 2}+\frac{1}{2} \operatorname{Tr}\left(D_{i} \Phi\right)^{\dagger} D_{i} \Phi+V(\Phi)\right]
$$

can be expressed in terms of profile functions as

$$
\begin{aligned}
\mathcal{H}= & 2 \pi \int r d r\left[\frac{1}{2} \frac{\left(\partial_{r} h(r)\right)^{2}}{g_{\mathrm{W}}^{2} r^{2}}\right. \\
& +v_{3}^{2}\left\{\left(\partial_{r} f(r)\right)^{2}+\frac{h(r)^{2} f(r)^{2}}{r^{2}}+\frac{1}{2}\left(\partial_{r} g(r)\right)^{2}\right\} \\
& \left.+\lambda_{1} v_{3}^{4}\left[2 f(r)^{2}+g(r)^{2}-3\right]^{2}+2 \lambda_{2} v_{3}^{4}\left[f(r)^{2}-g(r)^{2}\right]^{2}\right] .
\end{aligned}
$$

This is actually the Hamiltonian density along the $z$ axis. Since all our fields are independent of the $z$ coordinate we omit the $z$ integral. Let us rewrite the above Hamiltonian by defining $l=\frac{\lambda_{2}}{\lambda_{1}}, \lambda_{\rho}=\frac{\lambda_{1}}{g_{\mathrm{W}}^{2}}$, and $\rho^{2}=2 g_{\mathrm{W}}^{2} v_{3}^{2} r^{2}$ as

$$
\begin{aligned}
\mathcal{H}= & 2 \pi v_{3}^{2} \times \epsilon\left(\lambda_{\rho}, l\right), \\
\epsilon\left(\lambda_{\rho}, l\right)= & \int \rho d \rho\left[\frac{\left(\partial_{\rho} h(\rho)\right)^{2}}{\rho^{2}}\right. \\
& +\left\{\left(\partial_{\rho} f(\rho)\right)^{2}+\frac{h(\rho)^{2} f(\rho)^{2}}{\rho^{2}}+\frac{1}{2}\left(\partial_{\rho} g(\rho)\right)^{2}\right\} \\
& \left.+\frac{\lambda_{\rho}}{2}\left[\left\{2 f(\rho)^{2}+g(\rho)^{2}-3\right\}^{2}+2 l\left[f(\rho)^{2}-g(\rho)^{2}\right]^{2}\right]\right] .
\end{aligned}
$$

The equations of motion can be read off as

$$
\begin{aligned}
& -\rho \partial_{\rho}\left[\frac{\partial_{\rho} h(\rho)}{\rho}\right]+f(\rho)^{2} h(\rho)=0, \\
& -\frac{1}{\rho} \partial_{\rho}\left[\rho \partial_{\rho} f(\rho)\right]+\frac{h(\rho)^{2} f(\rho)}{\rho^{2}}+2 \lambda_{\rho}\left[(2+l) f(\rho)^{2}\right. \\
& \left.+(1-l) g(\rho)^{2}-3\right] f(\rho)=0, \\
& -\frac{1}{\rho} \partial_{\rho}\left[\rho \partial_{\rho} g(\rho)\right]+2 \lambda_{\rho}\left[2(1-l) f(\rho)^{2}\right. \\
& \left.+(1+2 l) g(\rho)^{2}-3\right] g(\rho)=0 .
\end{aligned}
$$

The tension of the vortex can be computed by inserting the profile function into Eq. (42) and integrating over the $(x, y)$ plane. For the values of $\lambda_{1}=0.2, g_{\mathrm{W}}=0.63$ we find $\epsilon\left(\lambda_{\rho}\right) \simeq 2$. In Fig. 2, we show the profile functions for the cases of $l=0.5,1,5$. In the figure, we also plot the energy density as a function of $\rho$ for the case of $l=5$.
As we know, the VEV of $\Phi_{v}=v_{3} \mathbf{1}_{3 \times 3}$ preserves the $S U(2)_{\mathrm{V}}$ custodial symmetry. However the vortex ansatz in Eq. (36) breaks spontaneously the custodial $S U(2)_{\mathrm{V}}$ symmetry to a $U(1)$ subgroup inside the vortex due to the existence of two different profile behaviors, $f(r)$ and $g(r)$, inside the vortex core. This generates infinite degenerate solutions which can be parametrized by an element on $S^{2} \simeq S U(2)_{\mathrm{V}} / U(1)$. We may write a generic solution by a global $S U(2)_{\mathrm{V}}$ transformation as

$$
\begin{aligned}
\Phi_{\mathrm{vortex}}\left(r, \theta, \xi^{\alpha}\right) & =v_{3} G\left(\xi^{\alpha}\right)\left(\begin{array}{ccc}
e^{i \theta} f(r) & 0 & 0 \\
0 & g(r) & 0 \\
0 & 0 & e^{-i \theta} f(r)
\end{array}\right) G^{\dagger}\left(\xi^{a}\right), \\
W_{i}\left(r, \xi^{\alpha}\right) & =-\frac{\epsilon_{i j} x^{j}}{r^{2}}(1+h(r)) \hat{\mathbf{n}}\left(\xi^{\alpha}\right),
\end{aligned}
$$

where $\hat{\mathbf{n}}(\zeta)$ is a unit vector oriented along a generic point on $S U(2)_{\mathrm{V}} / U(1) \simeq S^{2}$ defined as

$$
\hat{\mathbf{n}}\left(\xi^{\alpha}\right)=G\left(\xi^{\alpha}\right) T^{3} G^{\dagger}\left(\xi^{\alpha}\right)=\hat{n}^{\alpha} T^{\alpha} .
$$

Here $G\left(\xi^{\alpha}\right)$ is an element in the coset $S^{2} \simeq S U(2)_{\mathrm{V}} / U(1)$, and $\xi^{\alpha}$ are the moduli parametrizing $S^{2}$.

These degenerated solutions can be varied slowly along the $z$ axis with time without changing the profile functions. So if we integrate the profile functions we end up with an effective action which is a nonlinear sigma model defined on a two-dimensional world sheet (here $t-z$ plane) where fields are parametrized by the moduli parameters $\xi^{\alpha}$.

\section{B. Topological $Z$ strings and $W$ strings in the presence of the $U(1)_{\mathrm{Y}}$ gauge interaction}

In the above discussion, we have discussed the symmetries of potential, which is the same as the case where there is no $B_{\mu}$ interaction or $g_{\mathrm{Y}}=0$. In this case all gauge fields are massive so we neglected their interactions. So we find massless NG modes on the vortex. However, in reality $g_{\mathrm{Y}} \neq 0$ and this breaks custodial symmetry explicitly; the NG modes are lifted to become pseudo-NG modes, and consequently most of the non-Abelian vortices become unstable. The same phenomenon was first found for nonAbelian vortices in dense QCD $[86,87]$ and later applied to supersymmetric QCD [101].

In this subsection we discuss the construction of vortices in the presence of the $B_{\mu}$ gauge field. The kinetic term of the scalar field is given as $\mathcal{D}_{\mu} \Phi=$ $\left(\partial_{\mu}-i g_{\mathrm{W}} W_{\mu}^{a} T^{a}\right) \Phi+i g_{\mathrm{Y}} \Phi B_{\mu} T^{3}$. So naturally there is a chance for the vortex flux to share the $B_{\mu}$ fields. As we know from Eq. (44) the general vortex solution in the absence of the $B_{\mu}$ field can be written with the flux directed along the unit vector $\hat{n}$ living on a sphere $S^{2} \simeq S U(2)_{\mathrm{V}} / U(1)$. However, as it can be seen from the expression of covariant derivative in Eq. (8), the covariant 


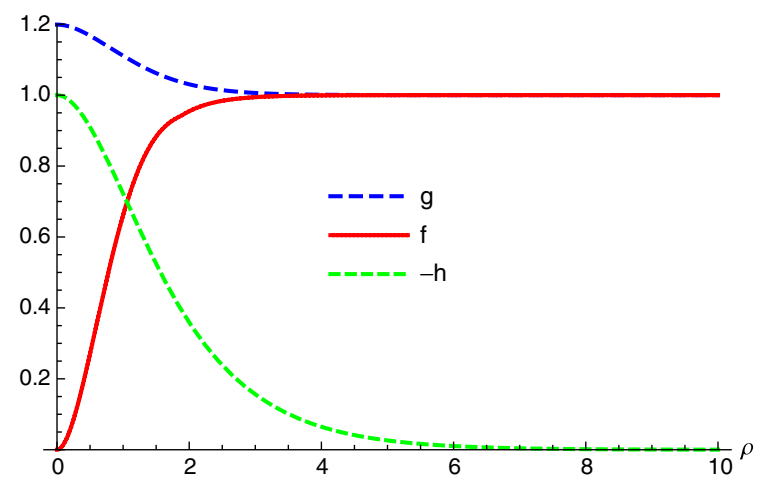

(a)

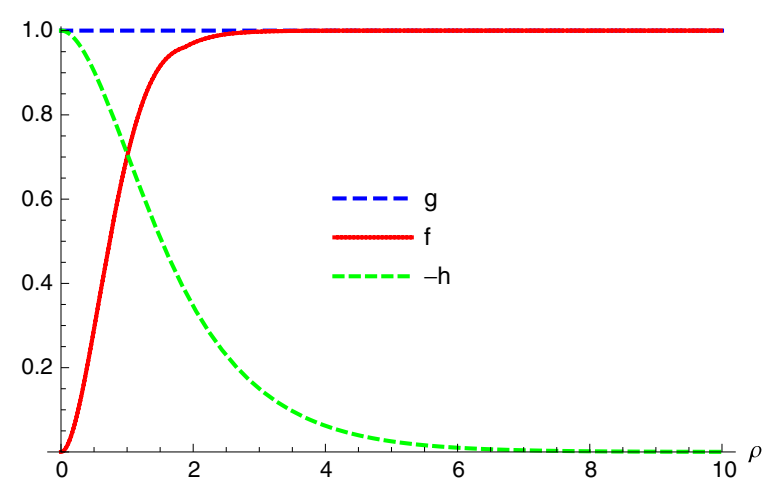

(c)

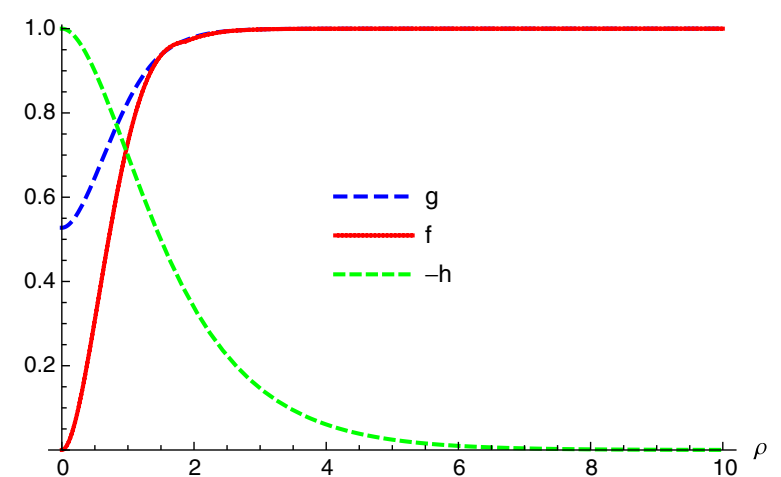

(b)

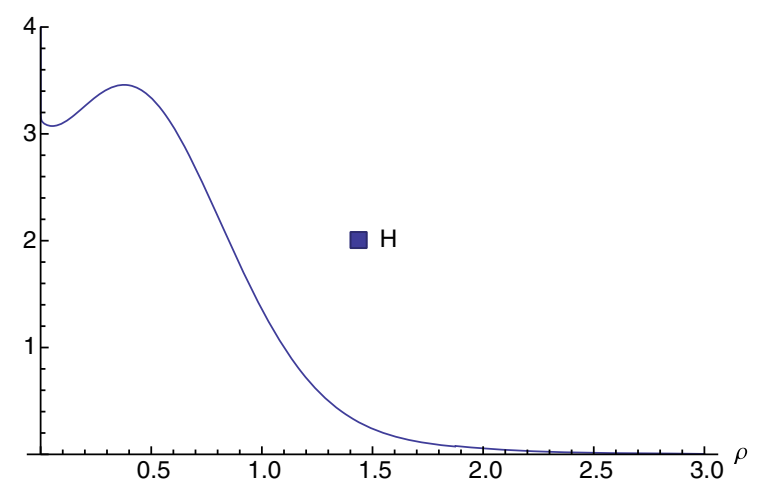

(d)

FIG. 2. This figure represents the plots of non-Abelian vortices discussed in Sec. IV. In this computation we used $\lambda_{1}=0.2, g_{\mathrm{W}}=0.63$. We choose $l=0.5$ for Fig. (a), $l=5$ for Fig. (b), and $l=1$ for Fig. (c). In Fig. (d) the energy density is plotted for the $l=5$ case.

derivative does not transform covariantly under the global transformation along generic direction on $S^{2}$ since the last term breaks the degeneracy. However, there exist degenerate solutions on a subspace which consist of the north and south pole of the sphere. The equator circle also gives degenerate $W$-string solutions, but their tension is a little higher than the $Z$ strings (north and south pole). We discuss the different configurations as follows.

\section{The $Z$ strings}

The $Z$ strings are defined on the north and south poles of the moduli space $S^{2}$. At the north pole the scalar field ansatz is given as

$$
\Phi_{\text {vortex }}=v_{3}\left(\begin{array}{ccc}
f(r) e^{i \theta} & 0 & 0 \\
0 & g(r) & 0 \\
0 & 0 & f(r) e^{-i \theta}
\end{array}\right) \text {, }
$$

with the boundary condition $f(0)=0, f(\infty)=1$, $g^{\prime}(0)=0, g(\infty)=1$. Now by solving large distance condition $\mathcal{D}_{i} \Phi \stackrel{r \rightarrow \infty}{\longrightarrow} 0$, We may find the gauge field ansatz as

$$
\begin{aligned}
Z_{i} & =\cos \theta_{\mathrm{W}} W_{i}^{3}-\sin \theta_{\mathrm{W}} B_{i}=-\frac{\epsilon_{i j} x^{j}}{g_{\mathrm{Z}} r^{2}}(1+h(r)), \\
h(0) & =-1, \quad h(\infty)=0,
\end{aligned}
$$

where $\cos \theta_{\mathrm{W}}=\frac{g_{\mathrm{W}}}{\sqrt{g_{\mathrm{W}}^{2}+g_{\mathrm{Y}}^{2}}}$ and $\sin \theta_{\mathrm{W}}=\frac{g_{\mathrm{Y}}}{\sqrt{g_{\mathrm{W}}^{2}+g_{\mathrm{Y}}^{2}}}$. The difference with Eq. (36) in the expression of the ansatz is that in Eq. (47) the coupling const $g_{\mathrm{W}}$ is replaced by $g_{\mathrm{Z}}=$ $\sqrt{g_{\mathrm{W}}^{2}+g_{\mathrm{Y}}^{2}}$.

Similarly, at the south pole the scalar field configuration is given as

$$
\Phi_{\mathrm{vortex}}=v_{3}\left(\begin{array}{ccc}
f(r) e^{-i \theta} & 0 & 0 \\
0 & g(r) & 0 \\
0 & 0 & f(r) e^{i \theta}
\end{array}\right) .
$$

The gauge field solution is the same as Eq. (47) with a negative sign in front.

Since the vortex flux is completely determined by the flux of the $Z_{\mu}$ field, these vortices can be called topological $Z$ strings. The tension of the $Z$ string can be computed the same way as we did in Sec. IVA with $g_{\mathrm{W}}$ replaced by $g_{\mathrm{Z}}$. We find that the tension of the $Z$ string is smaller than the case of $g_{\mathrm{Y}}=0$ by about $5 \%$. 


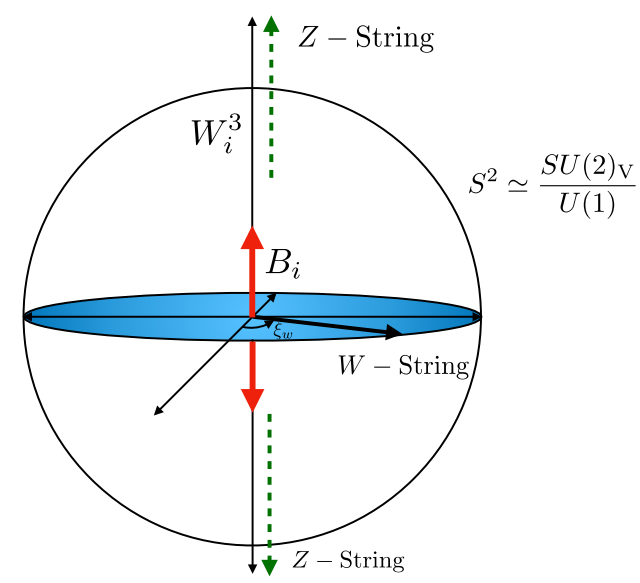

FIG. 3. A schematic view of $Z$ and $W$ strings on the moduli space $S^{2}$.

\section{The $W$ strings}

The $W$ strings are defined on the equator circle on $S^{2}$. In this case, there is no flux sharing with the $B_{\mu}$ field. The scalar field configurations are defined as

$$
\begin{aligned}
\Phi_{\mathrm{W}}\left(r, \theta, \xi_{\mathrm{W}}\right) & =G\left(\xi_{\mathrm{W}}\right) \Phi(r, \theta, 0) G^{\dagger}\left(\xi_{\mathrm{W}}\right), \\
G\left(\xi_{\mathrm{W}}\right) & =\left(\begin{array}{ccc}
\frac{e^{-i \xi_{\mathrm{W}}}}{\sqrt{2}} & 1 & \frac{e^{-i \xi_{\mathrm{W}}}}{\sqrt{2}} \\
1 & 0 & -1 \\
\frac{e^{i \xi_{\mathrm{W}}}}{\sqrt{2}} & -1 & \frac{e^{i \xi_{\mathrm{W}}}}{\sqrt{2}}
\end{array}\right),
\end{aligned}
$$

where $\Phi(r, \theta, 0)=\operatorname{diag}\left(f(r) e^{i \theta}, g(r), f(r) e^{-i \theta}\right)$. The $W_{\mu}$ gauge field configurations are given in the orthogonal direction to the $Z$ strings as

$$
\begin{aligned}
\left(\begin{array}{c}
W_{i}^{1} \\
W_{i}^{2}
\end{array}\right) & =-\frac{2 \epsilon_{i j} x^{j}}{g_{\mathrm{W}} r^{2}}(1+h(r))\left(\begin{array}{c}
\cos \xi_{\mathrm{W}} \\
\sin \xi_{\mathrm{W}}
\end{array}\right), \\
W_{i}^{3} & =0 .
\end{aligned}
$$

Here $\xi_{\mathrm{W}}$ is parametrizing the degenerate solutions along the equator circle. The $W$-string solutions are constructed by global transformation of the solution written in Eq. (36). Therefore, the energy is same as the vortex constructed in the case of $g_{\mathrm{Y}}=0$, which means their tension is little higher (about 5\%) than the $Z$ strings (north and south pole). Therefore, the $W$ strings are energetically unstable. In Fig. 3, we show the schematic picture of $Z$ and $W$ strings on the moduli space of $S^{2}$.

\section{NON-ABELIAN DOMAIN WALLS BOUNDED BY NON-ABELIAN VORTICES}

In Sec. III we have constructed a stable domain wall in the GM model in the second symmetry breaking $\mathrm{H}_{3} \rightarrow \mathrm{H}_{2}$ with a hierarchical symmetry breaking $\mathrm{G} \rightarrow \mathrm{H}_{3} \rightarrow \mathrm{H}_{2}$. In the last section, we have constructed a vortex configuration in the first symmetry breaking $G \rightarrow H_{3}$, as described in Eq. (2). In this section, we consider the most general case, the coexistence of domain walls and vortices in the full symmetry breaking. In the first subsection, we observe a behavior of a doublet around a vortex for preparation of the subsequent subsections. In the second subsection, we show that the general configuration is a partly global and partly local vortex in the vanishing limit of the interaction between the doublet and triplets. In the third subsection, we show that it is a non-Abelian domain wall bounded by a non-Abelian vortex where $S^{2}$ moduli match at the junction line if we turn off the $U(1)_{\mathrm{Y}}$ gauge coupling, while it is a $Z$ wall bounded by a $Z$ string if we turn on the $U(1)_{\mathrm{Y}}$ gauge coupling. Finally, in the fourth subsection, we calculate the quantum decay rate of a domain wall by quantum tunneling by creating a hole bounded by a closed vortex line.

\section{A. Behavior of a doublet encircling around a vortex}

We show in this subsection that when the doublet field acquires a VEV a pathology happens. To this end, we first investigate what happens when a doublet field encircles a vortex. We note that the large distance behavior of a vortex configuration along the $z$ axis can be written as

$\Phi_{\text {vortex }}(r=\infty, \theta)=\Omega_{3}(\theta) \Phi_{\text {vortex }}(r=\infty, \theta=0)$,

where $\Omega_{3}$ is a holonomy acting on the triplet fields around a vortex, given by

$$
\Omega_{3}(\theta)=P e^{i \int_{0}^{\theta} W \cdot d l}=e^{i \theta T^{3}} .
$$

Here, we note that $\Omega_{3}$ is single valued as usual, $\Omega_{3}(2 \pi)=\mathbf{1}_{3 \times 3}$.

On the other hand, when a field $\eta(x)$ in a doublet representation encircles a vortex, it receives a gauge transformation

$$
\eta(r=\infty, \theta)=\Omega_{2}(\theta) \eta(r=\infty, \theta=0),
$$

where $\Omega_{2}$ is a holonomy acting on the doublet, given by

$$
\Omega_{2}(\theta)=P e^{i \int_{0}^{\theta} W \cdot d l}=e^{i \theta \tau^{3}} .
$$

In this case, it has a nontrivial holonomy when it encircles a vortex

$$
\Omega(2 \pi)=-\mathbf{1}_{2 \times 2},
$$

and consequently

$$
\eta(\theta=2 \pi)=-\eta(\theta=0) .
$$

Therefore, the doublet field cannot become single valued around a vortex, and so it has a nontrivial AharanovBohm phase. 
This brings us a pathology; the doublet field may not be allowed to acquire a VEV, and consequently the SM symmetry breaking could not occur in the presence of a vortex. This puzzle can be solved in two ways. One can make a vortex to a global vortex or one can create a domain wall. The former happens when the interaction between the doublet and triplet is negligible, namely, when $\lambda_{4}$ is small in Eq. (9). The latter happens when the interaction term with $\lambda_{4}$ is relevant. In the following subsections, we discuss these two cases separately.

\section{B. A composite of the global-local vortex configuration}

Here we discuss a vortex configuration which develops after the second symmetry breaking in a special circumstance. We switch off the interaction term between the doublet and triplets, i.e., $\lambda_{4}=0$ and also hypercharge $g_{\mathrm{Y}}=0$. In this case, the triplets and doublet fields interact via the $S U(2)_{\mathrm{L}}$ gauge interaction only. Since they are in the absence of the $\lambda_{4}$ term, the right actions on the doublet and triplets are independent, which we denote $S U(2)_{\mathrm{R}_{1}}$ and $S U(2)_{\mathrm{R}_{2}}$, respectively. So in this case, we start with full symmetry breaking group as $G\left(R_{1}, R_{2}\right)=$ $S U(2)_{\mathrm{L}} \times S U(2)_{\mathrm{R}_{1}} \times S U(2)_{\mathrm{R}_{2}}$. Now we set our vacuum expectation values of the fields as before as $\Phi_{v}=v_{3} \mathbf{1}_{3 \times 3}$ and $\Psi_{v}=v_{2} \mathbf{1}_{2 \times 2}$; this breaks $G\left(R_{1}, R_{2}\right)$ to the diagonal group $H_{2}\left(R_{1}, R_{2}\right)=\mathbb{Z}_{2} \times S U(2)_{\mathrm{L}+\mathrm{R}_{1}+\mathrm{R}_{2}}$. The full symmetry breaking is discussed in Appendix in detail. The existence of a new vortex solution can be understood, if we note that the vacuum manifold

$$
\frac{G\left(R_{1}, R_{2}\right)}{H_{2}\left(R_{1}, R_{2}\right)}=\frac{S U(2)_{\mathrm{L}} \times S U(2)_{\mathrm{R}_{1}} \times S U(2)_{\mathrm{R}_{2}}}{\mathbb{Z}_{2} \times S U(2)_{\mathrm{L}+\mathrm{R}_{1}+\mathrm{R}_{2}}}
$$

allows the first homotopy group

$$
\pi_{1}\left(\frac{G\left(R_{1}, R_{2}\right)}{H_{2}\left(R_{1}, R_{2}\right)}\right)=\mathbb{Z}_{2}
$$

The $\mathbb{Z}_{2}$ factor of $H_{2}$ contains the element $(-1,1,-1)$ which would be responsible for our new vortex solution and this would keep the doublet field single valued. This is because rotation around an existing flux tube generates a negative sign where the existence of a global rotation in $S U(2)_{\mathrm{R}_{2}}$ generates another negative sign to compensate the other. In order to construct a vortex solution, let us define our doublet ansatz as

$$
\Psi_{\text {vortex }}(r, \theta)=v_{2} \psi(r) \exp \left(i \theta \sigma^{3}\right)
$$

with the boundary conditions for the profile function $\psi$ given by

$$
\psi(0)=0, \quad \psi(\infty)=1 .
$$

With this ansatz for the doublet field, we use the ansatz for the triplet fields and gauge field given in Eq. (36). The large distance behavior of the doublet field can be expressed as

$$
\Psi_{\mathrm{vortex}}(\infty, \theta)=P e^{i \int_{0}^{\theta} W \cdot d l} \Psi_{v} e^{i \theta \frac{\sigma^{3}}{2}}
$$

From this expression it is now clear that the full loop of $\pi_{1}\left(G / H_{2}\right)$ has two contributions. One is from an $S U(2)_{\mathrm{L}}$ gauge transformation accompanied with a gauge flux and the other is a global transformation of $S U(2)_{\mathrm{R}_{2}}$. Therefore, our vortex is a half local and half global vortex with a magnetic flux.

By using the vortex ansatz in Eqs. (36) and (61), we may rewrite the Hamiltonian, by defining $l=\frac{\lambda_{2}}{\lambda_{1}}, \lambda_{\rho}=\frac{\lambda_{1}}{g_{\mathrm{W}}^{2}}$, $\tilde{\lambda}_{3}=\frac{\lambda_{3}}{g_{\mathrm{W}}^{2}}$, and $\rho^{2}=2 g_{\mathrm{W}}^{2} v_{3}^{2} r^{2}, v=\frac{v_{2}}{v_{3}}$, as

$$
\begin{aligned}
\mathcal{H}= & 2 \pi v_{3}^{2} \int \rho d \rho\left[\frac{\left(\partial_{\rho} h(\rho)\right)^{2}}{\rho^{2}}+\left\{\left(\partial_{\rho} f(\rho)\right)^{2}+\frac{h^{2} f(\rho)^{2}}{\rho^{2}}+\frac{1}{2}\left(\partial_{\rho} g(\rho)\right)^{2}\right\}+\frac{\lambda_{\rho}}{2}\left[\left\{2 f(\rho)^{2}+g(\rho)^{2}-3\right\}^{2}+2 l\left[f(\rho)^{2}-g(\rho)^{2}\right]^{2}\right]\right] \\
& +\pi v_{2}^{2} \int \rho d \rho\left[\left(\partial_{\rho} \psi(\rho)\right)^{2}+\frac{(1-h)^{2} \psi(\rho)^{2}}{4 \rho^{2}}+\tilde{\lambda}_{3} v^{2}\left[\psi(\rho)^{2}-1\right]^{2}\right] .
\end{aligned}
$$

The equations of motion can be read off as

$$
\begin{array}{r}
-\rho \partial_{\rho}\left[\frac{\partial_{\rho} h(\rho)}{\rho}\right]+f(\rho)^{2} h(\rho)+\frac{v^{2}}{8}(h(\rho)-1) \psi(\rho)^{2}=0, \\
-\frac{1}{\rho} \partial_{\rho}\left[\rho \partial_{\rho} f(\rho)\right]+\frac{h(\rho)^{2} f(\rho)}{\rho^{2}}+2 \lambda_{\rho}\left[(2+l) f(\rho)^{2}+(1-l) g(\rho)^{2}-3\right] f(\rho)=0, \\
-\frac{1}{\rho} \partial_{\rho}\left[\rho \partial_{\rho} g(\rho)\right]+2 \lambda_{\rho}\left[2(1-l) f(\rho)^{2}+(1+2 l) g(\rho)^{2}-3\right] g(\rho)=0, \\
-\frac{1}{\rho} \partial_{\rho}\left[\rho \partial_{\rho} \psi(\rho)\right]+\frac{(1-h(\rho))^{2} \psi(\rho)}{4 \rho^{2}}+2 \tilde{\lambda}_{3} v^{2}\left[\psi^{2}-1\right] \psi=0 .
\end{array}
$$

A numerical solution is shown in Fig. 4. 


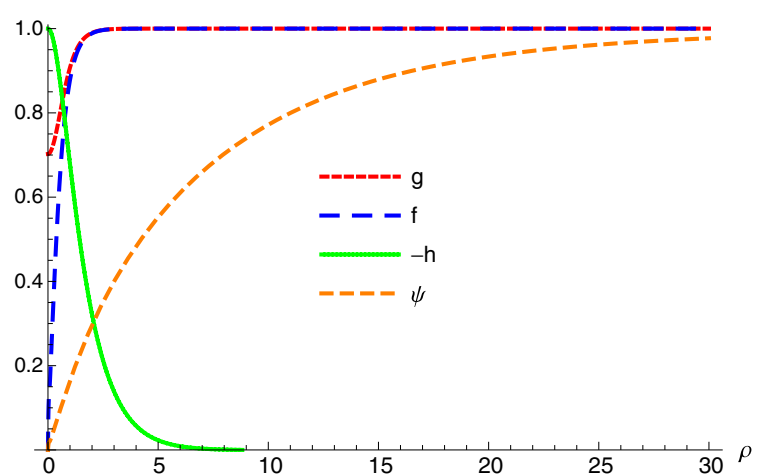

(a)

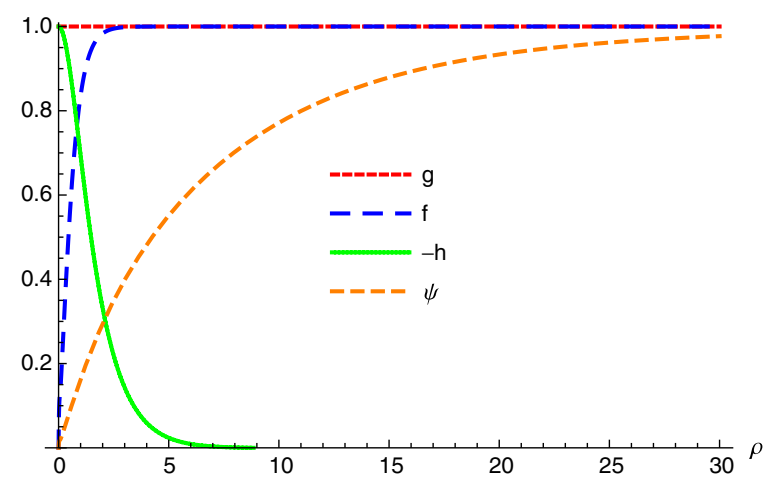

(c)

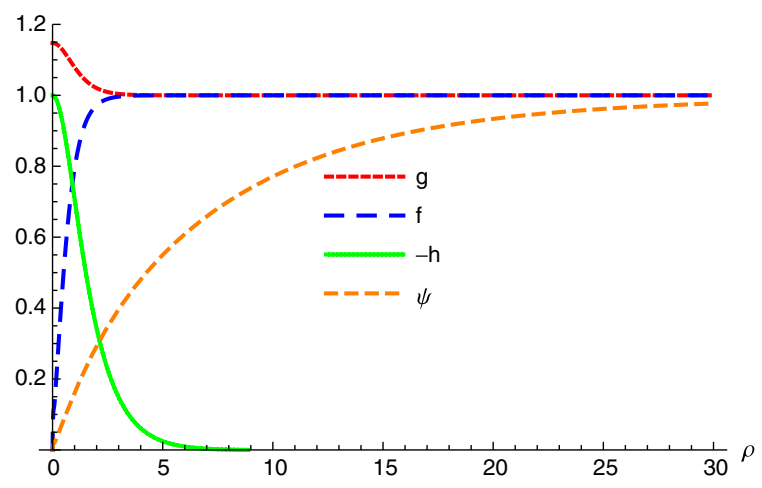

(b)

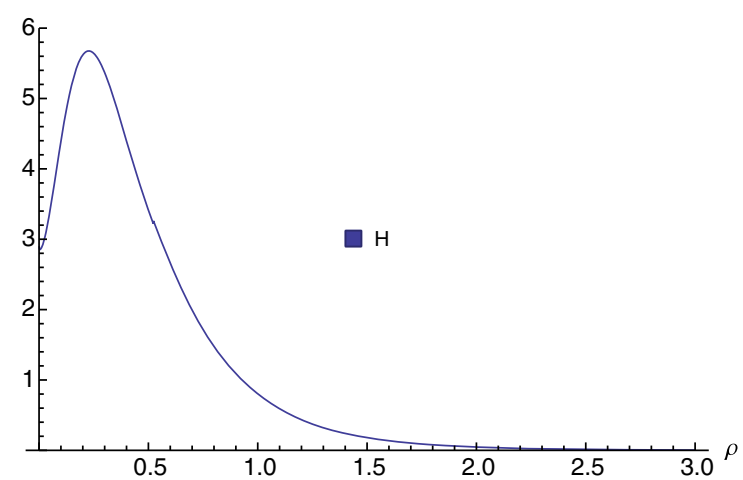

(d)

FIG. 4. The plots of the profile functions of a global vortex described in Sec. V B. In this calculation, we have used $\lambda_{1}=0.2, \lambda_{3}=0.2, g_{\mathrm{W}}=0.63$. We choose $l=5$ for panel (a), $l=0.5$ for panel (b), and $l=1$ for panel (c). In panel (d), the energy density is plotted for $l=5$.

\section{Domain wall bounded by a vortex}

In the last subsection we have discussed the case in which the $\lambda_{4}$ interaction term between the doublet and triplets is negligible so that the global symmetries acting on the doublet and triplets become independent and so the global symmetry is enhanced. In this subsection we consider the case in which the $\lambda_{4}$ term is relevant so that the global symmetries acting on the doublet and triplets are locked: $S U(2)_{\mathrm{R}_{1}}=S U(2)_{\mathrm{R}_{2}}$. We show that in this case the global vortex in the last subsection transforms to be a vortex-domain wall composite. We start with $g_{\mathrm{Y}}=0$ just to understand the orientational zero modes. The effect of hypercharge, i.e., $g_{\mathrm{Y}} \neq 0$, is discussed later.

In Sec. VA we have discussed the puzzle that the VEV of $\Psi$ might become multivalued around the vortex. Now we show that this problem can be cured by a creation of a domain wall in the doublet so that the total configuration becomes a vortex attached by a domain wall. As we discussed in Sec. III, at the second symmetry breaking $H_{3}=\mathbb{Z}_{2} \times S U(2)_{\mathrm{V}}$ is broken down to $S U(2)_{\mathrm{V}}$. This gives $\pi_{0}\left(H_{3} / H_{2}\right)=\mathbb{Z}_{2}$, which confirms the existence of the domain wall solution. To find a domain wall attached to the vortex, we start with the ansatz
$\Psi(x)_{\mathrm{dw}-\mathrm{vortex}}=\frac{v_{2}}{\sqrt{2}} \psi(r)\left(\begin{array}{cc}e^{\left.i \frac{\phi(\theta)+\xi(\theta)}{2}\right]} & 0 \\ 0 & e^{-i\left[\frac{\phi(\theta)+\xi(\theta)}{2}\right]}\end{array}\right)$,

where $\xi(\theta)$ and $\phi(\theta)$, both of which change from 0 to $2 \pi$ when one goes around a vortex, are contributions from the gauge transformation and the global $S U(2)_{\mathrm{R}}$ transformation, respectively. In this subsection, we study a large distance behavior of the system. The vortex solution at large distance behaves as

$$
\Phi_{\text {vortex }} \sim v_{3} \exp \left[i \xi(\theta) T^{3}\right], \quad W_{i} \sim \frac{\partial_{i} \xi(\theta)}{g_{\mathrm{W}}} T^{3} .
$$

We insert the field configurations in Eqs. (64) and (65) into each term of the potential term in Eq. (9),

$$
\begin{aligned}
V(\Phi, \Psi)= & \lambda_{3}\left(\operatorname{Tr} \Psi \Psi^{\dagger} \Psi-v_{2}^{2}\right)^{2} \\
& +\lambda_{4}\left(\operatorname{Tr} \Psi \Psi^{\dagger} \Psi \Phi^{\dagger} \Phi\right. \\
& \left.-2 \operatorname{Tr}\left(\Psi^{\dagger} \tau^{a} \Psi \tau^{b}\right) \operatorname{Tr}\left(\Phi^{\dagger} T^{a} \Phi T^{b}\right)\right),
\end{aligned}
$$

to yield 


$$
\begin{aligned}
\operatorname{Tr}\left(\Psi^{\dagger} \tau^{a} \Psi \tau^{b}\right) & =\frac{v_{2}^{2}}{4} \psi^{2} R(\tau)_{a b}, \\
R(\tau) & =\left(\begin{array}{ccc}
\cos (\phi+\xi) & -\sin (\phi+\xi) & 0 \\
\sin (\phi+\xi) & \cos (\phi+\xi) & 0 \\
0 & 0 & 1
\end{array}\right),
\end{aligned}
$$

$\operatorname{Tr}\left(\Phi^{\dagger} T^{a} \Phi T^{b}\right)=2 v_{3}^{2} R(T)_{a b}$,

$$
R(T)=\left(\begin{array}{ccc}
\cos \xi & -\sin \xi & 0 \\
\sin \xi & \cos \xi & 0 \\
0 & 0 & 1
\end{array}\right) .
$$

We thus obtain

$$
V(\psi, \phi)=v_{2}^{2}\left[\lambda_{3} v_{2}^{2}\left(\psi(\theta)^{2}-1\right)^{2}+\mu^{2} \psi^{2}(1-\cos \phi(\theta))\right],
$$

where we have defined $\mu^{2}=2 \lambda_{4} v_{3}^{2}$ as before in Eq. (29). This potential is the same as what was found in Eq. (29), although the argument here is the angle $\theta$ around the vortex while it was one spatial direction $x$ in Eq. (29). The ground state is given by $\psi=1, \phi=2 n \pi$. As we have found in Sec. III this potential gives a sine-Gordon domain wall solution. This domain wall is attached to the infinitely long vortex along the $z$ axis centered at the origin, as schematically shown in Fig. 5(a). When encircling around the vortex, the doublet field changes sign while passing through the domain wall placed at $\theta=\theta_{c}$. Therefore, the existence of the domain wall solves the problem that the doublet might become multivalued.

In our hierarchy symmetry breaking, the width of the vortex $\delta_{\text {vortex }} \sim\left(v_{3} \sqrt{\lambda_{1}}\right)^{-1}$ is much smaller than the wall width $\delta_{\mathrm{dw}} \sim \mu^{-1}$.

We have shown that domain walls are bounded by $\mathbb{Z}_{2}$ strings described in Sec. IV. One thing one should point out here is that our strings are not global strings but are flux tubes. This construction is in contrast to the axionic stringdomain wall composite, in which strings are global strings.

Let us discuss here the orientational zero modes and effect of hypercharge, i.e., $g_{\mathrm{Y}} \neq 0$. The computations done above do not depend on the orientation of the vortex and domain wall. They can be oriented together in generic directions on $S^{2} \simeq S U(2)_{\mathrm{V}} / U(1)$ since the $\lambda_{4}$ term keeps the triplet and the doublet in the same direction. Oscillations of the zero modes would supposedly flow from the vortex world sheet to the domain wall surface and vice versa. Actual dynamics would be described by effective action of the NG modes, which is the $O(3)$ model with a boundary.

When $g_{\mathrm{Y}} \neq 0$ the NG modes are lifted. So at lowest energy, we have only a $Z$-string-domain wall.

\section{Quantum decay of a domain wall}

The domain wall construction discussed in this paper is not stable after full symmetry breaking $\pi_{0}\left(G / H_{2}\right)=0$. So according to Kibble [105], a hole would be created locally due to local thermal or quantum fluctuations. This hole is bounded by a closed vortex string as schematically shown in Fig. 5(b). A hole smaller than some critical scale $R_{c}$ will be destroyed. However, there can be some hole creation with length scale more than $R_{c}$. Then this hole would start growing and the wall would become unstable. The decay rate can be computed using the method described in Refs. $[23,105]$. The decay probability at zero temperature is given as $\Gamma \sim e^{-S}$ where $S$ is the Euclidean action of the bounce solution corresponding to the tunneling process. In this case $S$ is given as

$$
S=4 \pi R^{2} T_{\text {vortex }}-\frac{4}{3} \pi R^{3} T_{\mathrm{dw}} .
$$

Here $T_{\text {vortex }}=2 \pi v_{3}^{2} \epsilon(\epsilon \sim 2)$ is the vortex energy per unit length as defined in Eq. (42) and $T_{\mathrm{dw}}=4 \mu v_{2}^{2}$ is the energy of the domain wall per unit area, defined in Eq. (29). So $R_{c}=\frac{2 T_{\text {vorex }}}{T_{\mathrm{dw}}}$, and

$$
S_{c}=\frac{16 \pi}{3}\left(\frac{T_{\mathrm{vortex}}^{3}}{T_{\mathrm{dw}}^{2}}\right)=\frac{4 \pi^{4}}{3 \lambda_{4}}\left(\frac{v_{3}}{v_{2}}\right)^{4} \epsilon^{3} .
$$

In the case of $v_{3} \gg v_{2}$ and $\lambda_{4}<1, \Gamma$ must be a small number. So domain walls can be locally stable at zero temperature. However, this is a minimal estimation of the decay rate because this computation is valid when $S$ is very large so that other interactions can be neglected [106]. So when $v_{3}$ is close to $v_{2}$ this analysis may not be very

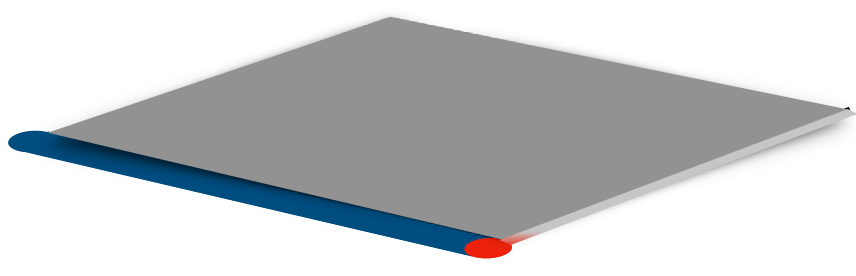

(a)

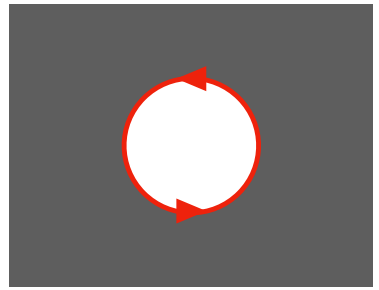

(b)

FIG. 5. The schematic diagrams of the (a) domain wall bounded by flux tube (b) hole creation in the domain wall. 
practical. We also did not take into account the finite temperature effect. The interaction with other fields and finite temperature effects may change this probability and would be discussed elsewhere.

\section{SUMMARY AND DISCUSSIONS}

In this paper, we have discussed topological defects in the GM model. This model contains three Higgs triplets in addition to the usual Higgs doublet. We studied the spontaneous breaking of $G=S U(2)_{\mathrm{L}} \times S U(2)_{\mathrm{R}}$ in two stages, namely a hierarchical symmetry breaking $G \rightarrow$ $\mathrm{H}_{3} \rightarrow \mathrm{H}_{2}$ first triggered by the triplets followed by the doublet subsequently. The order parameter manifold has nontrivial homotopy groups $\pi_{1}\left(G / H_{3}\right)=\mathbb{Z}_{2}$ and $\pi_{0}\left(H_{3} / H_{2}\right)=\mathbb{Z}_{2}$ supporting a $\mathbb{Z}_{2}$ vortex and a domain wall, respectively. We have first solved the vortex profile functions numerically for the case of an axially symmetric infinitely long vortex in the decoupling limit of the $U(1)_{\mathrm{Y}}$ gauge field. In this case, the custodial $S U(2)_{\mathrm{V}}$ is spontaneously broken inside the vortex core generating the $S^{2} \mathrm{NG}$ modes localized around the vortex core. These modes correspond to a non-Abelian magnetic flux confined inside the vortex core. When the $U(1)_{\mathrm{Y}}$ gauge coupling is taken into account, the $S U(2)_{\mathrm{V}}$ custodial symmetry is explicitly broken, the NG modes become pseudo-NG modes, and the $S^{2}$ moduli space is lifted, leaving a stable $Z$ string and an unstable $W$ string as solutions. We find that a $Z$ string has a $5 \%$ lower tension than that of a $W$ string or a non-Abelian string [of zero $U(1)$ gauge coupling]. All vortices including $W$ strings fall into a topologically stable $Z$ string, in contrast to the $\mathrm{SM}$ in which $Z$ strings are nontopological and are unstable in the realistic parameter region. We then have discussed the vortex-domain wall complex, in which the $S^{2}$ moduli of both the vortex and domain wall match at the junction line. The vortex stable in the first symmetry breaking is attached by a domain wall appearing in the second symmetry breaking, and consequently domain walls can decay through quantum tunneling by creating a hole bounded by a closed vortex line. We have calculated the decay rate at zero temperature, which is found to be small.

Several discussions are addressed here. In this paper, we have discussed only the Higgs sector. If we include the fermion sector, there are several interesting physics. First, vortices [107] and domain walls [108] would have fermion zero modes around their cores, as an electroweak $Z$ string in the SM [35-43] and a non-Abelian vortex in dense QCD $[109,110]$. For the former, it was argued that fermion zero modes may destabilize $Z$ strings, but in our case strings are stable (at the first symmetry breaking) because they are topological. For the latter, these fermion zero modes interact with NG modes [91], and so a similar thing happens in our case.

Second, fermions scattering off a non-Abelian vortex may receive an Aharanov-Bohm phase, as the cases of an electroweak $Z$ string $[111,112]$ and a non-Abelian vortex in dense QCD [90] (see also Ref. [113] for the same situation in supersymmetric QCD). All together, the exchange of multiple vortices with fermion zero modes may have nontrivial non-Abelian statistics like the case of dense QCD [114-117].

The interaction of the electromagnetic waves and topological defects found in this paper may be important for a possibility of searches for these objects, such as cosmic microwave backgrounds. The interaction of the electromagnetic waves and a non-Abelian vortex through charged zero modes localized around the vortex was studied in dense QCD, in which case a vortex lattice is shown to behave as a polarizer [118]. In our case, the interaction with a domain wall through the charged zero modes localized around it must be the most important possibility. The $Z$ string (or $Z$-string-domain wall composite) does not interact with electromagnetic gauge field $A_{\mu}$ at low energies. Since NG modes are massive the high-energy electromagnetic waves can excite NG modes, which interact with $A_{\mu}$. So in this sense the $Z$ string (-domain wall composite) could be considered as a "gray-matter" element.

Electroweak baryogenesis by electroweak strings [32] does not work in the SM [34]. This problem may be rescued since our $Z$ strings are topologically stable in the first symmetry breaking. If there is a long enough period between the first and second symmetry breakings there could be enough baryogenesis.

Gravitational waves from the decay of domain walls and vortices give important signature of this scenario [119]. Depending on the tension of domain walls and the temperature when they annihilate, the amount of gravitational waves emitted from those could be significant enough to be detected by ongoing and future experimental searches. A detailed study of the spectrum of gravitational waves in the GM model is made elsewhere.

Other than vortices and domain walls, there may exist stable monopoles (instantons) and skyrmions as composite states, in contrast to the SM. Stable monopoles may exist as a kink on a $Z$ string since $S^{2}$ moduli are lifted due to the $U(1)_{\mathrm{Y}}$ gauge coupling, leaving two points, the north and south poles, corresponding to two $Z$ strings. In this case, a monopole is attached by two $Z$ strings on both sides and so is stable, different from electroweak Nambu monopoles. Instantons may exist as lumps inside a vortex [120], but they may be unstable because of the potential induced from the $U(1)_{\mathrm{Y}}$ gauge coupling. On the other hand, skyrmions may exist as lumps inside a domain wall (called domain wall skyrmions) [63,64,121,122] (see also [59]) whose effective theory should be an $O(3)$ sigma model.

\section{ACKNOWLEDGMENTS}

We thank Michikazu Kobayashi for helping us with numerical coding. This work is supported by the Ministry of Education, Culture, Sports, Science (MEXT)-Supported Program for the Strategic Research Foundation at Private 
Universities "Topological Science" (Grant No. S1511006). C. C. acknowledges support as an International Research Fellow of the Japan Society for the Promotion of Science (JSPS) (Grant No. 16F16322). M. K. acknowledges support from MEXT Grants-in-Aid for Scientific Research on Innovative Areas (Grant No. 25105011). The work of M. N. is supported in part by JSPS Grant-in-Aid for Scientific Research (KAKENHI Grant No. 16H03984), and by a Grant-in-Aid for Scientific Research on Innovative Areas "Topological Materials Science" (KAKENHI Grant No. 15H05855) from the MEXT of Japan.

\section{APPENDIX: SYMMETRY OF GLOBAL VORTICES}

Here we discuss the symmetry and symmetry breaking in the absence of the interaction between the doublet and triplets, i.e., $\lambda_{4}=0$ and $g_{\mathrm{Y}}=0$. In this case the triplets and doublet fields interact only through the $S U(2)_{\mathrm{L}}$ gauge interaction, since in this case the right symmetries act independently on the triplets and doublet. We denote the right action groups on the triplets and doublet by $S U(2)_{\mathrm{R}_{1}}$ and $S U(2)_{\mathrm{R}_{2}}$, respectively. So in this case the full symmetry group is given as

$$
G\left(R_{1}, R_{2}\right)=S U(2)_{\mathrm{L}} \times S U(2)_{\mathrm{R}_{1}} \times S U(2)_{\mathrm{R}_{2}} .
$$

Now the VEV of the triplet fields $\Phi_{v}=v_{3} \mathbf{1}_{3 \times 3}$ breaks $G\left(R_{1}, R_{2}\right)$ to (see footnote 4)

$$
\begin{aligned}
H_{3}\left(R_{1}, R_{2}\right) & =\left(\mathbb{Z}_{2}\right)_{-\mathrm{L}+\mathrm{R}_{1}} \times S U(2)_{\mathrm{L}+\mathrm{R}_{1}} \times S U(2)_{\mathrm{R}_{2}}, \\
\left(\mathbb{Z}_{2}\right)_{-\mathrm{L}+\mathrm{R}_{1}} & =\{(1,1,1),(-1,1,1)\} .
\end{aligned}
$$

The first two entries in the elements of $\mathbb{Z}_{2}$ are arising from the center of $S U(2)_{\mathrm{L}}$ and $S U(2)_{\mathrm{R}_{1}}$. This $\mathbb{Z}_{2}$ is unbroken because the center is identified in the triplet representation.
Now let us discuss the second symmetry breaking by $\Psi_{v}=v_{2} \mathbf{1}_{2 \times 2}$, which breaks $H_{3}$ further into

$$
\begin{aligned}
H_{2} & =\left(\mathbb{Z}_{2}\right)_{-\mathrm{L}+\mathrm{R}_{1}-\mathrm{R}_{2}} \times S U(2)_{\mathrm{L}+\mathrm{R}_{1}+\mathrm{R}_{2}}, \\
\left(\mathbb{Z}_{2}\right)_{-\mathrm{L}+\mathrm{R}_{1}-\mathrm{R}_{2}} & =\{(1,1,1),(-1,1,-1)\} .
\end{aligned}
$$

Note that $\left(\mathbb{Z}_{2}\right)_{-\mathrm{L}+\mathrm{R}_{1}-\mathrm{R}_{2}}$ is different from $\left(\mathbb{Z}_{2}\right)_{-\mathrm{L}+\mathrm{R}_{1}}$ in $H_{3}$.

To understand this let us write down the unbroken elements of the full center of $G\left(R_{1}, R_{2}\right)$, which is $\mathbb{Z}_{2} \times \mathbb{Z}_{2} \times \mathbb{Z}_{2}$, as

$$
\{(1,1,1),(-1,-1,-1),(1,-1,1),(-1,1,-1)\} .
$$

All the nontrivial elements of this group have order 2. We may rewrite these elements as an internal direct product of two $\mathbb{Z}_{2}$ subgroups as

$\mathbb{Z}_{2} \times \mathbb{Z}_{2}=\{(1,1,1),(-1,-1,-1)\} \times\{(1,1,1),(-1,1,-1)\}$.

This is an internal direct product in which the identity element is shared. The first $\mathbb{Z}_{2}$ factor is the center of the diagonal subgroup $S U(2)_{\mathrm{L}+\mathrm{R}_{1}+\mathrm{R}_{2}}$, while the second factor is $\left(\mathbb{Z}_{2}\right)_{-\mathrm{L}+\mathrm{R}_{1}-\mathrm{R}_{2}}$ in Eq. (A3).

The existence of the new vortex solution can be understood if we see the fundamental group of the full symmetry breaking as

$$
\begin{aligned}
\pi_{1}\left(\frac{G\left(R_{1}, R_{2}\right)}{H_{2}\left(R_{1}, R_{2}\right)}\right) & =\pi_{1}\left(\frac{S U(2)_{\mathrm{L}} \times S U(2)_{\mathrm{R}_{1}} \times S U(2)_{\mathrm{R}_{2}}}{\mathbb{Z}_{2} \times S U(2)_{\mathrm{L}+\mathrm{R}_{1}+\mathrm{R}_{2}}}\right) \\
& =\mathbb{Z}_{2} .
\end{aligned}
$$

[1] G. Aad et al. (ATLAS Collaboration), Observation of a new particle in the search for the standard model Higgs boson with the ATLAS detector at the LHC, Phys. Lett. B 716, 1 (2012).

[2] S. Chatrchyan et al. (CMS Collaboration), Observation of a new boson at a mass of $125 \mathrm{GeV}$ with the CMS experiment at the LHC, Phys. Lett. B 716, 30 (2012).

[3] H. Georgi and M. Machacek, Doubly charged Higgs bosons, Nucl. Phys. B262, 463 (1985).

[4] M. S. Chanowitz and M. Golden, Higgs boson triplets with $M(W)=M(Z) \cos \theta \omega$, Phys. Lett. 165B, 105 (1985).

[5] S. Blasi, S. De Curtis, and K. Yagyu, Effects of custodial symmetry breaking in the Georgi-Machacek model at high energies, Phys. Rev. D 96, 015001 (2017).
[6] S. I. Godunov, M. I. Vysotsky, and E. V. Zhemchugov, Double Higgs production at LHC, seesaw type II and Georgi-Machacek model, J. Exp. Theor. Phys. 120, 369 (2015).

[7] C. W. Chiang, A. L. Kuo, and T. Yamada, Searches of exotic Higgs bosons in general mass spectra of the GeorgiMachacek model at the LHC, J. High Energy Phys. 01 (2016) 120.

[8] J. Chang, C. R. Chen, and C. W. Chiang, Higgs boson pair productions in the Georgi-Machacek model at the LHC, J. High Energy Phys. 03 (2017) 137.

[9] C. W. Chiang, S. Kanemura, and K. Yagyu, Phenomenology of the Georgi-Machacek model at future electronpositron colliders, Phys. Rev. D 93, 055002 (2016). 
[10] Y. Zhang, H. Sun, X. Luo, and W. Zhang, Searching for the heavy charged custodial fiveplet Higgs boson in the Georgi-Machacek model at the International Linear Collider, Phys. Rev. D 95, 115022 (2017).

[11] B. Li, Z. L. Han, and Y. Liao, Higgs production at future $e^{+} e^{-}$colliders in the Georgi-Machacek model, J. High Energy Phys. 02 (2018) 007.

[12] S. Kanemura, K. Tsumura, K. Yagyu, and H. Yokoya, Fingerprinting nonminimal Higgs sectors, Phys. Rev. D 90, 075001 (2014).

[13] L. Cort, M. Garcia, and M. Quiros, Supersymmetric custodial triplets, Phys. Rev. D 88, 075010 (2013).

[14] H. E. Logan and V. Rentala, All the generalized GeorgiMachacek models, Phys. Rev. D 92, 075011 (2015).

[15] R. Campbell, S. Godfrey, H. E. Logan, and A. Poulin, Real singlet scalar dark matter extension of the GeorgiMachacek model, Phys. Rev. D 95, 016005 (2017).

[16] C. W. Chiang and T. Yamada, Electroweak phase transition in the Georgi-Machacek model, Phys. Lett. B 735, 295 (2014).

[17] T. W. B. Kibble, Topology of cosmic domains and strings, J. Phys. A 9, 1387 (1976).

[18] W. H. Zurek, Cosmological experiments in superfluid helium?, Nature (London) 317, 505 (1985).

[19] H. B. Nielsen and P. Olesen, Vortex line models for dual strings, Nucl. Phys. B61, 45 (1973).

[20] A. A. Abrikosov, On the magnetic properties of superconductors of the second group, Zh. Eksp. Teor. Fiz. 32, 1442 (1957); [Sov. Phys. JETP 5, 1174 (1957)].

[21] T. W. B. Kibble, G. Lazarides, and Q. Shafi, Strings in SO (10), Phys. Lett. 113B, 237 (1982).

[22] M. B. Hindmarsh and T. W. B. Kibble, Cosmic strings, Rep. Prog. Phys. 58, 477 (1995).

[23] A. Vilenkin and E. P.S. Shellard, Cosmic Strings and other Topological Defects, Cambridge Monographs on Mathematical Physics (Cambridge University Press, Cambridge, 2000).

[24] Y. Nambu, Stringlike configurations in the WeinbergSalam theory, Nucl. Phys. B130, 505 (1977).

[25] T. Vachaspati, Vortex Solutions in the Weinberg-Salam Model, Phys. Rev. Lett. 68, 1977 (1992); Erratum, 69, 216 (1992).

[26] T. Vachaspati, Electroweak strings, Nucl. Phys. B397, 648 (1993).

[27] R. Holman, S. Hsu, T. Vachaspati, and R. Watkins, Metastable cosmic strings in realistic models, Phys. Rev. D 46, 5352 (1992).

[28] M. James, L. Perivolaropoulos, and T. Vachaspati, Detailed stability analysis of electroweak strings, Nucl. Phys. B395, 534 (1993).

[29] A. Achucarro and T. Vachaspati, Semilocal and electroweak strings, Phys. Rep. 327, 347 (2000);

[30] M. Eto, K. Konishi, M. Nitta, and Y. Ookouchi, Brane realization of Nambu monopoles and electroweak strings, Phys. Rev. D 87, 045006 (2013).

[31] T. Vachaspati and A. Achucarro, Semilocal cosmic strings, Phys. Rev. D 44, 3067 (1991).

[32] R. H. Brandenberger and A. C. Davis, Electroweak baryogenesis with electroweak strings, Phys. Lett. B 308, 79 (1993).
[33] M. Barriola, Electroweak strings that produce baryons, Phys. Rev. D 51, R300 (1995).

[34] M. Nagasawa and J. Yokoyama, Are Nontopological Strings Produced at the Electroweak Phase Transition?, Phys. Rev. Lett. 77, 2166 (1996).

[35] T. Vachaspati and R. Watkins, Bound states can stabilize electroweak strings, Phys. Lett. B 318, 163 (1993).

[36] M. A. Earnshaw and W. B. Perkins, Stability of an electroweak string with a fermion condensate, Phys. Lett. B 328, 337 (1994).

[37] J. Garriga and T. Vachaspati, Zero modes on linked strings, Nucl. Phys. B438, 161 (1995).

[38] J. M. Moreno, D. H. Oaknin, and M. Quiros, Fermions on the electroweak string, Phys. Lett. B 347, 332 (1995).

[39] S. G. Naculich, Fermions Destabilize Electroweak Strings, Phys. Rev. Lett. 75, 998 (1995).

[40] H. Liu and T. Vachaspati, Perturbed electroweak strings and fermion zero modes, Nucl. Phys. B470, 176 (1996).

[41] G. D. Starkman, D. Stojkovic, and T. Vachaspati, Neutrino zero modes on electroweak strings, Phys. Rev. D 63, 085011 (2001).

[42] G. Starkman, D. Stojkovic, and T. Vachaspati, Zero modes of fermions with a general mass matrix, Phys. Rev. D 65, 065003 (2002).

[43] N. Graham, M. Quandt, and H. Weigel, Fermion energies in the background of a cosmic string, Phys. Rev. D 84, 025017 (2011).

[44] J. Preskill and A. Vilenkin, Decay of metastable topological defects, Phys. Rev. D 47, 2324 (1993).

[45] T. Vachaspati, Estimate of the Primordial Magnetic Field Helicity, Phys. Rev. Lett. 87, 251302 (2001).

[46] R. Poltis and D. Stojkovic, Can Primordial Magnetic Fields Seeded by Electroweak Strings Cause an Alignment of Quasar Axes on Cosmological Scales?, Phys. Rev. Lett. 105, 161301 (2010).

[47] F. R. Klinkhamer and N.S. Manton, A saddle point solution in the Weinberg-Salam theory, Phys. Rev. D 30, 2212 (1984).

[48] J. K. Perring and T. H. R. Skyrme, A model unified field equation, Nucl. Phys. 31, 550 (1962).

[49] N. S. Manton and P. Sutcliffe, Topological Solitons (Cambridge University Press, Cambridge, 2004), p. 493.

[50] R. Rajaraman, Solitons and Instantons. An Introduction to Solitons and Instantons in Quantum Field Theory (North-Holland, Amsterdam, Netherlands, 1982), p. 409.

[51] A. V. Ustinov, Solitons in Josephson junctions, Physica (Amsterdam) 123D, 315329 (1998).

[52] T. Vachaspati, Kinks and Domain Walls: An Introduction to Classical and Quantum Solitons (Cambridge University Press, Cambridge, 2006).

[53] A. Vilenkin and A. E. Everett, Cosmic Strings and Domain Walls in Models with Goldstone and Pseudo-Goldstone Bosons, Phys. Rev. Lett. 48, 1867 (1982).

[54] A. E. Everett and A. Vilenkin, Left-right symmetric theories and vacuum domain walls and strings, Nucl. Phys. B207, 43 (1982).

[55] Y. B. Zeldovich, I. Y. Kobzarev, and L. B. Okun, Cosmological consequences of the spontaneous breakdown of discrete symmetry, Zh. Eksp. Teor. Fiz. 67, 3 (1974); [Sov. Phys. JETP 40, 1 (1974)]. 
[56] D. Stojkovic, K. Freese, and G. D. Starkman, Holes in the walls: Primordial black holes as a solution to the cosmological domain wall problem, Phys. Rev. D 72, 045012 (2005).

[57] M. Kawasaki and K. Nakayama, Axions: Theory and cosmological role, Annu. Rev. Nucl. Part. Sci. 63, 69 (2013).

[58] M. Nitta, Non-Abelian sine-Gordon solitons, Nucl. Phys. B895, 288 (2015).

[59] M. Eto and M. Nitta, Non-Abelian sine-Gordon solitons: Correspondence between $S U(N)$ skyrmions and $\mathbb{C} P^{N-1}$ lumps, Phys. Rev. D 91, 085044 (2015).

[60] M. Nitta, Josephson junction of non-Abelian superconductors and non-Abelian Josephson vortices, Nucl. Phys. B899, 78 (2015).

[61] M. Nitta, Josephson instantons and Josephson monopoles in a non-Abelian Josephson junction, Phys. Rev. D 92 , 045010 (2015).

[62] A. Ritz, M. Shifman, and A. Vainshtein, Enhanced world volume supersymmetry and intersecting domain walls in $N=1$ SQCD, Phys. Rev. D 70, 095003 (2004).

[63] M. Nitta, Correspondence between skyrmions in $2+1$ and 3+ 1 dimensions, Phys. Rev. D 87, 025013 (2013).

[64] M. Nitta, Matryoshka skyrmions, Nucl. Phys. B872, 62 (2013).

[65] D. I. Olive and N. Turok, $Z_{2}$ vortex strings in grand unified theories, Phys. Lett. 117B, 193 (1982).

[66] M. Hindmarsh and T. W. B. Kibble, Beads on Strings, Phys. Rev. Lett. 55, 2398 (1985).

[67] M. A. C. Kneipp, Color superconductivity, Z(N) flux tubes, and monopole confinement in deformed $N=2 *$ superYang-Mills theories, Phys. Rev. D 69, 045007 (2004).

[68] V. Markov, A. Marshakov, and A. Yung, Non-Abelian vortices in $N=1 *$ gauge theory, Nucl. Phys. B709, 267 (2005).

[69] A. Hanany and D. Tong, Vortices, instantons, and branes, J. High Energy Phys. 07 (2003) 037.

[70] R. Auzzi, S. Bolognesi, J. Evslin, K. Konishi, and A. Yung, Non-Abelian superconductors: Vortices and confinement in $N=2$ SQCD, Nucl. Phys. B673, 187 (2003).

[71] R. Auzzi, S. Bolognesi, J. Evslin, and K. Konishi, NonAbelian monopoles and the vortices that confine them, Nucl. Phys. B686, 119 (2004).

[72] A. Hanany and D. Tong, Vortex strings and fourdimensional gauge dynamics, J. High Energy Phys. 04 (2004) 066.

[73] M. Shifman and A. Yung, Non-Abelian string junctions as confined monopoles, Phys. Rev. D 70, 045004 (2004).

[74] A. Gorsky, M. Shifman, and A. Yung, Non-Abelian Meissner effect in Yang-Mills theories at weak coupling, Phys. Rev. D 71, 045010 (2005).

[75] M. Eto, Y. Isozumi, M. Nitta, K. Ohashi, and N. Sakai, Moduli Space of Non-Abelian Vortices, Phys. Rev. Lett. 96, 161601 (2006).

[76] M. Eto, K. Konishi, G. Marmorini, M. Nitta, K. Ohashi, W. Vinci, and N. Yokoi, Non-Abelian vortices of higher winding numbers, Phys. Rev. D 74, 065021 (2006).

[77] M. Eto, K. Hashimoto, G. Marmorini, M. Nitta, K. Ohashi, and W. Vinci, Universal Reconnection of Non-Abelian Cosmic Strings, Phys. Rev. Lett. 98, 091602 (2007).
[78] M. Eto, Y. Isozumi, M. Nitta, K. Ohashi, and N. Sakai, Solitons in the Higgs phase: The moduli matrix approach, J. Phys. A 39, R315 (2006).

[79] A. P. Balachandran, S. Digal, and T. Matsuura, Semisuperfluid strings in high density QCD, Phys. Rev. D 73, 074009 (2006).

[80] E. Nakano, M. Nitta, and T. Matsuura, Non-Abelian strings in high density QCD: Zero modes and interactions, Phys. Rev. D 78, 045002 (2008).

[81] E. Nakano, M. Nitta, and T. Matsuura, Non-Abelian strings in hot or dense QCD, Prog. Theor. Phys. Suppl. 174, 254 (2008).

[82] M. Eto and M. Nitta, Color magnetic flux tubes in dense QCD, Phys. Rev. D 80, 125007 (2009).

[83] M. Eto, E. Nakano, and M. Nitta, Effective world-sheet theory of color magnetic flux tubes in dense QCD, Phys. Rev. D 80, 125011 (2009).

[84] M. Eto, M. Nitta, and N. Yamamoto, Instabilities of NonAbelian Vortices in Dense QCD, Phys. Rev. Lett. 104, 161601 (2010).

[85] Y. Hirono, T. Kanazawa, and M. Nitta, Topological interactions of non-Abelian vortices with quasiparticles in high density QCD, Phys. Rev. D 83, 085018 (2011).

[86] W. Vinci, M. Cipriani, and M. Nitta, Spontaneous magnetization through non-Abelian vortex formation in rotating dense quark matter, Phys. Rev. D 86, 085018 (2012).

[87] M. Cipriani, W. Vinci, and M. Nitta, Colorful boojums at the interface of a color superconductor, Phys. Rev. D 86, 121704 (2012).

[88] M. Eto, Y. Hirono, M. Nitta, and S. Yasui, Vortices and other topological solitons in dense quark matter, Prog. Theor. Exp. Phys. 2014, 12D01 (2014).

[89] M. Kobayashi, E. Nakano, and M. Nitta, Color magnetism in non-Abelian vortex matter, J. High Energy Phys. 06 (2014) 130.

[90] C. Chatterjee and M. Nitta, Aharonov-Bohm phase in high density quark matter, Phys. Rev. D 93, 065050 (2016).

[91] C. Chatterjee, M. Cipriani, and M. Nitta, Coupling between Majorana fermions and Nambu-Goldstone bosons inside a non-Abelian vortex in dense QCD, Phys. Rev. D 93, 065046 (2016).

[92] C. Chatterjee and M. Nitta, Low-energy effective world sheet theory of a non-Abelian vortex in high-density QCD revisited: A regular gauge construction, Phys. Rev. D 95, 085013 (2017).

[93] M. Eto, L. Ferretti, K. Konishi, G. Marmorini, M. Nitta, K. Ohashi, W. Vinci, and N. Yokoi, Non-Abelian duality from vortex moduli: A dual model of color confinement, Nucl. Phys. B780, 161 (2007).

[94] M. Nitta and W. Vinci, Non-Abelian monopoles in the Higgs phase, Nucl. Phys. B848, 121 (2011).

[95] K. Konishi, Physics of non-Abelian confinement and the dual gauge symmetry: Many faces of flavor symmetry, Nucl. Phys. B, Proc. Suppl. 234, 333 (2013).

[96] M. Cipriani, D. Dorigoni, S. B. Gudnason, K. Konishi, and A. Michelini, Non-Abelian monopole-vortex complex, Phys. Rev. D 84, 045024 (2011).

[97] C. Chatterjee and K. Konishi, Monopole-vortex complex at large distances and non-Abelian duality, J. High Energy Phys. 09 (2014) 039. 
[98] C. Chatterjee and A. Lahiri, Flux dualization in broken SU(2), J. High Energy Phys. 02 (2010) 033.

[99] C. Chatterjee and A. Lahiri, Monopoles and flux strings from SU(2) adjoint scalars, J. High Energy Phys. 09 (2009) 010.

[100] M. Eto, M. Nitta, and N. Yamamoto, Confined monopoles induced by quantum effects in dense QCD, Phys. Rev. D 83, 085005 (2011).

[101] K. Konishi, M. Nitta, and W. Vinci, Supersymmetry breaking on gauged non-Abelian vortices, J. High Energy Phys. 09 (2012) 014.

[102] G. R. Dvali and G. Senjanovic, Topologically Stable Electroweak Flux Tubes, Phys. Rev. Lett. 71, 2376 (1993).

[103] M. Eto, M. Kurachi, and M. Nitta, Universal bound on the mass of the CP-odd Higgs in two Higgs doublet models, arXiv:1803.04662.

[104] M. Eto, M. Kurachi, and M. Nitta, Non-Abelian strings and domain walls in two Higgs doublet models, arXiv:1805 .07015 .

[105] T. W. B. Kibble, G. Lazarides, and Q. Shafi, Walls bounded by strings, Phys. Rev. D 26, 435 (1982).

[106] S. Weinberg, The Quantum Theory of Fields, Modern Applications Vol. 2 (Cambridge University Press, Cambridge, 1996), p. 464.E. J. Weinberg, Classical solutions in quantum field theories, Annu. Rev. Nucl. Part. Sci. 42, 177 (1992).

[107] R. Jackiw and P. Rossi, Zero modes of the vortex-fermion system, Nucl. Phys. B190, 681 (1981).

[108] R. Jackiw and C. Rebbi, Solitons with fermion number 1/2, Phys. Rev. D 13, 3398 (1976).

[109] S. Yasui, K. Itakura, and M. Nitta, Fermion structure of non-Abelian vortices in high density QCD, Phys. Rev. D 81, 105003 (2010).

[110] T. Fujiwara, T. Fukui, M. Nitta, and S. Yasui, Index theorem and Majorana zero modes along a non-Abelian vortex in a color superconductor, Phys. Rev. D 84, 076002 (2011).

[111] H. K. Lo, Scattering from electroweak strings, Phys. Rev. D 1995) 802 ,51).

[112] M. Nagasawa, Effect of quark scatter on baryogenesis by electroweak strings, Astropart. Phys. 5, 231 (1996).

[113] J. Evslin, K. Konishi, M. Nitta, K. Ohashi, and W. Vinci, Non-Abelian vortices with an Aharonov-Bohm effect, J. High Energy Phys. 01 (2014) 086.

[114] S. Yasui, K. Itakura, and M. Nitta, Majorana meets Coxeter: Non-Abelian Majorana fermions and nonAbelian statistics, Phys. Rev. B 83, 134518 (2011).

[115] Y. Hirono, S. Yasui, K. Itakura, and M. Nitta, Non-Abelian statistics of vortices with multiple Majorana fermions, Phys. Rev. B 86, 014508 (2012).

[116] S. Yasui, K. Itakura, and M. Nitta, Dirac returns: NonAbelian statistics of vortices with Dirac fermions, Nucl. Phys. B859, 261 (2012).

[117] S. Yasui, Y. Hirono, K. Itakura, and M. Nitta, Non-Abelian statistics of vortices with non-Abelian Dirac fermions, Phys. Rev. E 87, 052142 (2013).

[118] Y. Hirono and M. Nitta, Anisotropic Optical Response of Dense Quark Matter Under Rotation: Compact Stars as Cosmic Polarizers, Phys. Rev. Lett. 109, 062501 (2012).

[119] For a recent review see, e.g., K. Saikawa, A review of gravitational waves from cosmic domain walls, Universe 3, 40 (2017).

[120] M. Eto, Y. Isozumi, M. Nitta, K. Ohashi, and N. Sakai, Instantons in the Higgs phase, Phys. Rev. D 72, 025011 (2005).

[121] S. B. Gudnason and M. Nitta, Domain wall skyrmions, Phys. Rev. D 89, 085022 (2014).

[122] S. B. Gudnason and M. Nitta, Incarnations of skyrmions, Phys. Rev. D 90, 085007 (2014). 